\title{
Übersicht über die Sporenassoziationen (lepidophyta- bis pusilla-Zone) aus dem Unterkarbon der Bohrung Neuenkirchen 2/1973 (Insel Rügen, NE-Deutschland)
}

\author{
Gusti Burmann ${ }^{\top}$ \\ Mit 1 Abbildung, 5 Tabellen und 2 Anhängen
}

\begin{abstract}
Zusammenfassung
Es wird die Sporenzonierung für das Tournai (einschließlich der Grenzschichten) der Bohrung Neuenkirchen 2/73 auf der Insel Rügen (NE-Deutschland) vorgelegt - von der lepidophyta-Zone var. minor-Subzone des Tnla des obersten Devons bis zur pusilla-Zone des tieferen Visé. Das Profil wird verglichen mit der Zonenabfolge aus der Bohrung Wiek 4/70 (Burmann 1975). Zur lepidophyta-Zone liegen vergleichbare Daten aus dem Harz vor (Burmann 1976).

Die untersuchte Abfolge von Sporenzonen umfasst die lepidophyta-Zone mit der var. minor-Subzone (höheres Tn1a): PA (pusillites-asperitis; Tn1b); NRH (nitidus-rarituberculatus-hederatus; Tn1b-Tn2); ND (nitidus-distinctus; Tn2); RM (rarituberculatus-minutissimus; tieferes Tn3); CM (claviger-macra; höheres Tn3); Pu (pusilla; V1a+V1b; Obergrenze der Pu-Zone nicht erfasst). Die stratigraphische Einbindung der Sporenzonen erfolgte durch makrofaunistische Datierungen. Dic Pu-Zone ist in beiden Bohrungen untergliederbar durch einen Horizont mit Gloeocapsamorpha.
\end{abstract}

Schlüsselwörter: Rügen, Harz, Sporenzonierung, Devon/Karbon-Grenze, Tournai, Visé, lepidophyta-Zone, pusilla-Zone.

\begin{abstract}
Lower Carboniferous spore assemblages (lepidophyta zone to pusilla zone) in the Neuenkirchen 2/1973 borehole of Rügen Island, NE Germany

A spore zonation is given for the Tournaisian sequence of the Neuenkirchen $2 / 73$ borehole on the island of Rügen (NE Germany). The deposits range from the lepidophyta zone var. minor subzone of the uppermost Famennian (Tnla) to the pusilla zone of the basal Viséan. The results are compared with the miospore zonation of the borehole Wiek 4 (Burmann 1975), and comparable dates for the lepidophyta zone from the Harz mountains (Burmann 1976).

In ascending order the miospore zones examined are: lepidophyta zone with var. minor subzone (upper par1 of Tn1a); PA (pusillites-asperitis; Tn1b); NRH (nitidus-rarituberculatus-hederatus; Tn1b-Tn2); ND (nitidus-distinctus; Tn2); RM (rarituberculatus-minutissimus; lower part of Tn3); CM (claviger-macra; upper part of Tn3); Pu (pusilla; V1a+V1b; the upper boundary of the $\mathrm{Pu}$ zone is not determined). The stratigraphy of the spore zones is also compared with the macrofaunal data. The Pu zone in both boreholes is divided into two parts by a horizon with Gloeocapsamorpha.
\end{abstract}

Key words: Rügen, Harz, spore zonation, Devonian-Carboniferous boundary, Tournaisian, Viséan, lepidophyta zone, pusilla zone.

\section{Einleitung}

Die erste Sporenzonierung für das Unterkarbon von Rügen (Burmann 1975) umfasste den stratigraphischen Abschnitt von der lepidophytaZone, var. minor-Subzone (höheres Tn1a) des höchsten Oberdevons bis zur pusilla-Zone (erfasst im V1a und V1b) des tieferen Visé. Den Schwerpunkt bildete das Tournai mit seinen Grenzbereichen; die erarbeitete Zonierung beruhte auf den Daten aus der Bohrung Wiek 4 unter Einbeziehung von verallgemeinerten Da- ten auch anderer Rügen-Bohrungen, die im Detail nicht benannt werden durften. Wert wurde auf eine makrofaunistische Eichung der Sporenzonen gelegt. Danach erschien als stratigraphische Ergänzung zum Profil von Rügen (speziell des Devon/Karbon-Grenzbereiches) eine Arbeit über die lepidophyta-Zone (var. minor-Subzone und var. typica-Subzone) im Mittelharz (Burmann 1976).

Später haben Carson \& Clayton (1997) nach einer Bearbeitung der Unterkarbon-Profile der Bohrungen Rügen 2/1967 und Wiek 3/1968 eine

\footnotetext{
${ }^{1}$ Museum für Naturkunde Berlin, Institut für Paläontologie, Invalidenstr. 43, D-10115 Berlin, Germany. E-Mail: gusti.burmann@rz.hu-berlin.de Erhalten März 2001, angenommen Juli 2001
} 
Sporenzonierung für Rügen vorgestellt. in der nur das basale sowie das höchste Unterkarbon nicht erfasst waren. Jäger (2000) untersuchte die Unterkarbon-Frofile der Bohrungen Dranske 1/1968 und Dranske 2/1970 und folgte im Wesentlichen der Zonengliederung von Carson \& Clayton (1997) für Rügen.

Diese neuerın Bearbeitungen erfassen schwerpunktmäßig das Visé. Während Burmann in den Arbeiten der siebziger Jahre jeweils um eine regionale Einbindung der damals erst im Aufbau befindlichen Sporenzonierung in die makrofaunistische Gliecerung der Rügen-Bohrungen bemüht war. sind Carson. Clayton und Jäger offenbar einen and'ren methodischen Weg gegangen. Im Ergebnis gibt es einen Widerspruch der Daten dieser Bearbeiter zu den Angaben der lithostratigraphisch makrofaunistisch kontrollierten Gliederung des Unterkarbons von Rügen nach Hoffmann et al. (1975, 2002). Insbesondere betrifft das die abweichende Lage der Tournai/ Visé-Grenze (Carson \& Clayton 1997: 221). Ein weiteres Spezifikum von Rügen ist die erschwerte sporenstratigraphische Gliederungsmöglichkeit de's Visé. da sich die Sporenzonen des Visé Westıuropas nicht ohne weiteres übertragen lassen.

Dieser Kenntnisstand lässt sich durch den nachfolgenden Beilrag in drei wichtigen Punkten für das tiefere Unt rkarbon von Rügen erweitern:

- Die sporerstratigraphische Gliederung der Bohrungen Neuenkirchen 2 und Wiek 4 im Bereich der Tournai-Visé-Grenze wird dargestellt (CM-Żone und Pu-Zone). Dabei gibt es keinen Widerspruch (!) zwischen der Basis der Pu-Zoni: s. 1. (überregionale Anwendung) und der makrofaunistisch fixierten TournaiVisé-Grenze in beiden Bohrungen.

- Es werden arstmals aus der Bohrung Neuenkirchen 2 die Detailangaben zur lepidophyaZone aul $\mathrm{R}$ igen mitgeteilt. dic bereits in Burmann $(1975$ : 881, 883,884) in das Zonierungsschema von Rügen in allerdings verallgemeinerter Forn aufgenommen wurden. Die gleiche bolirungsbezogene Präzisierung ist nunmehr auch möglich für die PA- und NDZone, die 1975 ebenfalls bereits in das Übersichtsschema für Rügen (ohne Hinweis auf die He:kunft Bohrung Neuenkirchen 2) eingebunder waren.

- Die Kernmirsche eines cinzigen Bohrprofils (Neuenkirchen 2) liefern eine durchgehende sporenstratigraphische Abfolge vom höchsten Oberdevon (höheres Tnla) über das Tournai bis zum tiefren Visć (V1b).

\section{Material und Methode}

Dic Lage der Unterkarbon-Bohrpunkte auf der Insel Rügen ist in Abb. 1. dargestellt.

Die hisher sporenstratigraphisch untersuchten Unterkarhon-Profile gehören zu den Bohrungen Neuenkirchen 2/1973 Wiek +11970 und Sagard 1/1970 (Burmann unpubl. Berichte 1971. 1974: Burmann 1975 und diese Arbeit) sowie Rügen 2 1967 und Wiek 3/1968 (Carson unpubl. Diss. 1995; Carson \& Clayton 1997) und Dranske 1/1968 und Dranske 2/1970 (Jäger 2000). Alle genannten Bohrungen haben das Unterkarhon durchteuft.

Die Kernmarsch-bezogene Darstellung der Bohrung Neuenkirchen 2 mit den makrofaunistischen Datierungen (nach Dr. D. Weyer. unpubl.) und ausgewählten sporenstratigraphischen Daten sind als Übersicht der Tab. 1 zu entneh. men. Tab. 2 vergleicht dic Sporenzonen der Bohrunger Neuenkirchen 2 und Wiek 4 mit dem Zonenschema in Westeuropa. Taxonomische Daten sind der Taxa-Liste (Anhang 1) zu entnehmen. Im Anhang 2 sind dic Vergleichsgrundlager fur die laptophyta-Zone aus der Bohrung Neuenkirchen 2 aufgcfuihrt. Die Einzeldaten zu den Bohrungen Neucnkirchen 2 und Wiek 4 werden in Teil 2 vorgelegt.

Die Arbeiten zur Bohrung Neuenkirchen 2 wurden (zcitnah mit der Bearbeitung der Bohrung Wiek 4 und Sagard 1 in Wesentlichen 1974 ausgeführt, als sporenstratigraphische Zonierungen im Unterkarbon noch nicht auf ihre volle kor relative Aussage überprüft waren. Es wurde deshalb Wer gelegt auf eine regionale biostratigraphische Eichung. Makro. faunistische Einstufungen und sporenstratigraphische Dater der Bohrung Wiek 4 (Burmann 1975) werden zum Vergleich mit einbezogen. Die von Burmann (1975) publizierte Sporen. gliederung zum Unterkarbon von Rügen ging auf die ein malige Gelegenheit zurück, zum Karbonkongress in Moskat 1975 einen Beitrag publizieren zu dürfen. Trotzdem war es nur möglich. offiziell dic Bohrung Wiek 4 zu nennen unc nicht die Neuenkirchen 2, deren Daten aber im wesenticher Eingang in das publizierte Sporenzonenschema von Rüger fanden. da Letztere keine reine Forschungsbohrung war Dies wird nunmehr ergänzt durch die Publikation der Unter suchungsergebnisse zur Bohrung Neuenkirchen 2/1973.

In der Arbeit von Burmann (1975) muss untersehieder werden zwischen

- den Detaildaten in Form der taxonomischen Daten au einem Tn1b-Tn2 Kernmarsch (NRH-Zone) der Bohruns Wick 4. aus dem sämtliche fotographischen Abbildunger der Tafeln I-IX entstammten,

- der Sporenzonenabfolge der Bohrung Wiek 4 (NRH?hangende RM-. obere $\mathrm{CM}=\mathrm{Pu}-\mathrm{Z}$ one sensu lato) mit ma krofaunistischen Datierungen von $\mathrm{Tn} 1 \mathrm{~b}-\mathrm{Tn} 2 \mathrm{bis} \mathrm{V} 1 \mathrm{~b}$ und - der Zusammenstellung einer Sporenzonenabfolge für dic Insel Rügen. in die auljer den genannten sporenstratigra phischen Daten der Bohrung Wick 4 auch bereits zusätz liche Daten aus der Bohrung Ncuenkirchen 2 eingeflosser sind cohne dass der Fundort dieser ergänzenden Sporen zonen lepidophyta-var. minor-Subzone, PA-Zone, NRHND-. RM-Zone benannt werden durfte). Die stratigraphi sche Zuordnung für das Schema der Sporenzonen von Rü gen ist aber tabellarisch ausreichend klar dargestellt unc textlich formuliert.

Trotzdem wurde durch Jäger (1999: 88) etwas missverstan den: die PA-Zone ..... sowie dic darunterfolgende minor Subzone der lepidophytus-Zone sind nach heutigen Paralleli sierungen beide dem Tnla zuzurechnen, also dem oberster Deron". Die makrofaunistische Eichung der PA-Zone vor Rügen erfolgte aber am Tnlb. die Zone enthält keine lepido phira-Sporen mehr. Das Rügen-Profil der Unterkarbon-Spo renzonen setzt bei Jäger (1999: 91) in der Interpretations spalte 74 Burmann (1975) erst ab der NRH-Zone mi ungegliedertem Tn1b-Tn2 ein, was nicht korrekt dargestell ist: es beginnt bei Burmann (1975) und in dieser Arbeit mi der PA-Zone im Tnlb. Die Bohrung Neuenkirchen 2 ist dic einzige Bohrung mit der vollständigen Abfolge aller crkenn 


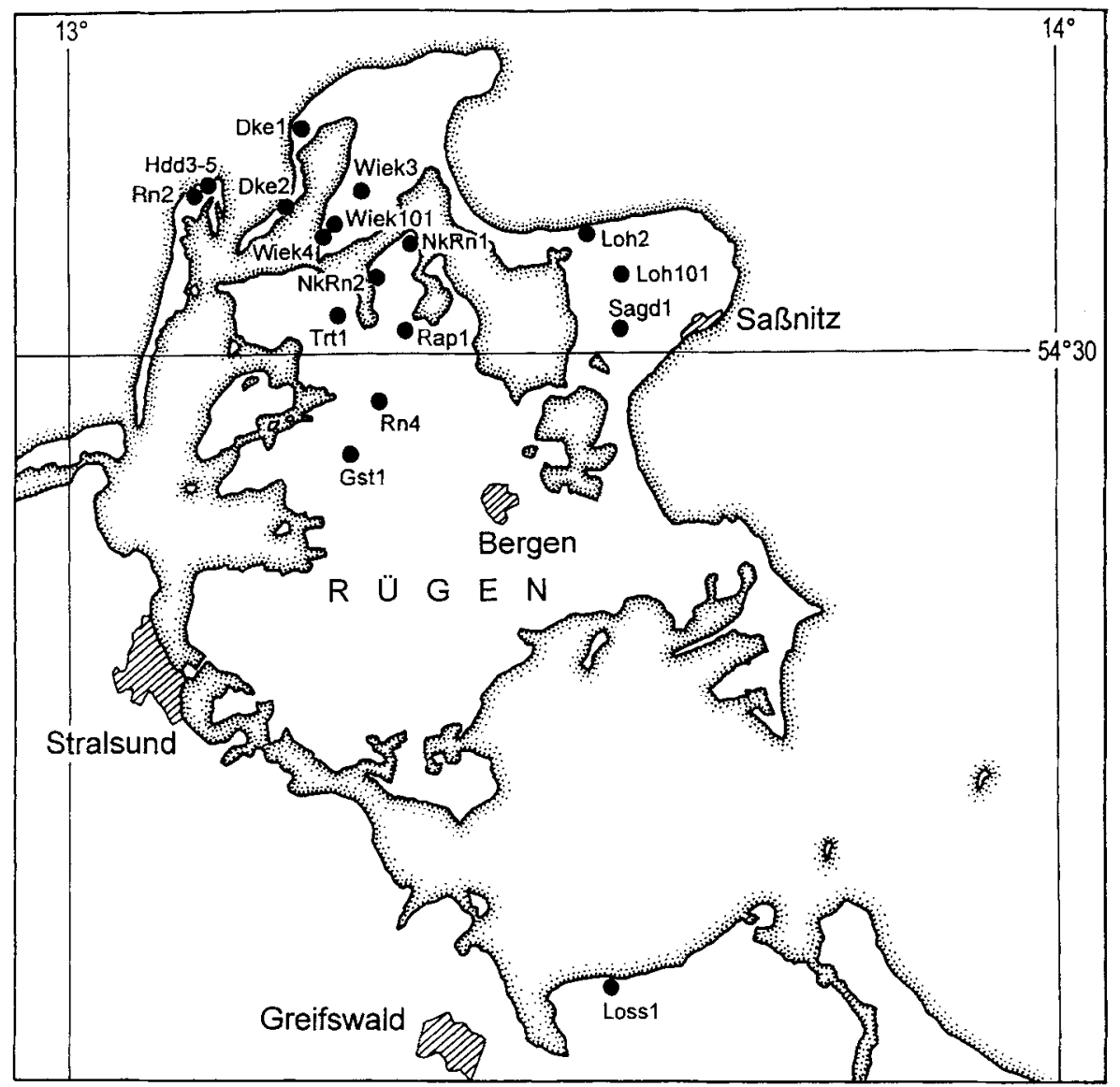

Abbildung 1. Bohrungen mit Unterkarbon auf der Insel Rügen. Im Text siehe Neuenkirchen 2 (NkRn2). Wiek 4. Sagard 1 und Bezug auf Rügen $2(\operatorname{Rn} 2)$, Wiek 3 .

baren Sporenzonen im Tournai von Rügen (beginnend mit der var. minor-Subzone der lepidophyta-Zone an der Devon/ Karbon-Grenze).

Die Schwierigkeit, das reale Erstauftreten von Lycospora pusilla zu erfassen, zwang zur Berücksichtigung anderer lokaler Hiliskriterien (Beispiel Gloeocapsamorpha-Maximum im V1a). Bei überregionalen Vergleichen ist es unerlässlich, dic von Autor zu Autor durchaus unterschiedlichen Kriterien der Zonendefinitionen und der Korrelation zu benennen.

\section{Sporenstratigraphische Gliederung auf Rügen (Tournai und Grenzschichten)}

Die in der Bohrung Neuenkirchen 2 ausgegliederten Sporenzonen sind in Tab. 1 und 2 aufgeführt. Das Profil widerspiegelt, trotz der Einschränkung durch die begrenzten Kernmärsche (Tab. 1), eine vollständige Abfolge von Sporenassoziationen vom höchsten Famenne bis zum basalen Visé, wie sie sonst auf Rügen (kernmarschbedingt) nicht erfasst wurde: lepidophyta var. minor-Subzone (höheres Tn1a); PA(pusillites-asperitis)-Zone (Tn1b); NRH(nitidus-rarituberculatus-hederatus)Zone (Tn1b-Tn2); ND(nitidus-distinctus)-Zone (Tn2); RM(rarituberculatus-minutissimus-Zone (tieferes Tn3); CM(claviger-macra)-Zone (unterer Teil, ohne Lycospora pusilla; höheres Tn3); CM-Zone (oberer Teil, mit seltenen Lycospora pusilla; regionale Anwendung auch bei fehlen- dem Nachweis von L. pusilla; V1a; basale $\mathrm{Pu}$ Zone sensu lato) mit Einschaltung des Gloeocapsamorpha-Horizontes (V1a) der Pu-Zone sensu lato; höherer Pu-Zonenanteil im V1(V?1b). Die Obergrenze der Pu-Zone ist in der Bohrung Neuenkirchen 2 durch fehlende Kernstrecken nicht erfasst.

Zum Vergleich steht die Sporenzonen-Gliederung der Bohrung Wiek 4 (Burmann 1975 und Burmann unpubl. 1971) zur Verfügung; im Prinzip ähneln sich die Abfolgen beider Aufschlüsse (Tab. 3).

Die in der Bohrung Wiek 4 nicht belegten Bereiche (Zonen ND und PA, lepidophyta var. minor-Subzone) sind durch dort fehlende Kernstrecken bedingt. Kann die NRH-Zone in beiden Bohrungen gut korreliert werden, so ist in der Wiek 4 die Äquivalenz zu der ND- und RM-Zone weniger deutlich ausgeprägt, wahrscheinlich durch die Lage der Kernmärsche.

Die CM-Zone ist in der Wiek 4 in ihrer typischen Ausprägung kernmarschbedingt ebenfalls nicht erfasst: nur der basale Übergang von der CM- zur RM-Zone ist wahrscheinlich gekernt worden. Der sporenstratigraphische Zusammenhang lässt sich wegen der Kernlücken nur vermuten, da die Abfolge im Gegensatz zur Neuenkirchen 2 unzureichend erfasst ist. 
Für die Pu-Zone stellte sich der Nachweis eines spezifitichen algenführenden Niveaus (Gloeocapsamorpha-Horizont) als wichtiges re- gionales Korrelationskriterium im V1a beider Bohrungen heraus. Die Lage der Pu-Zonenbasis mit dem seltenen Einsetzen von Lycospora pusil-

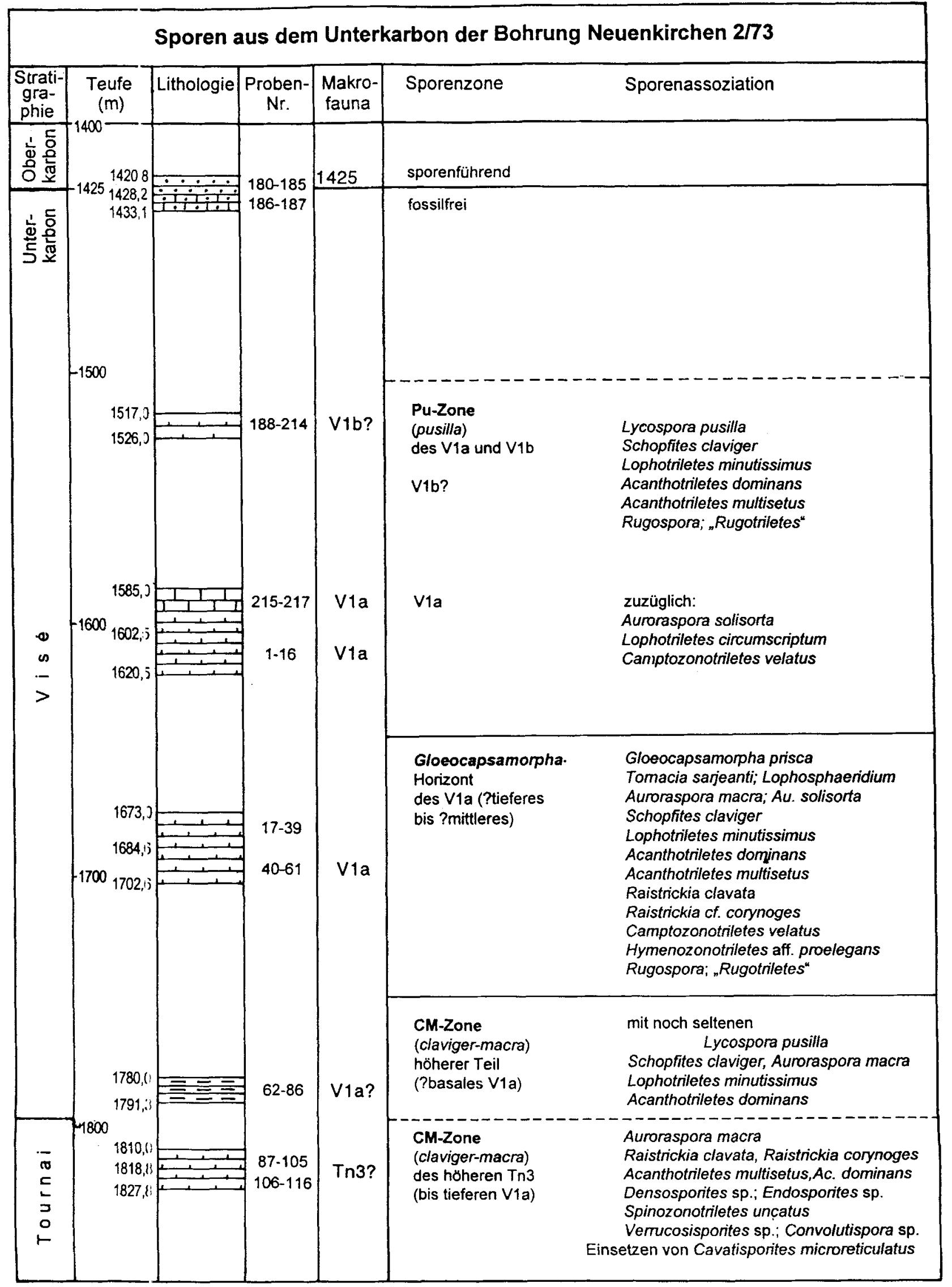




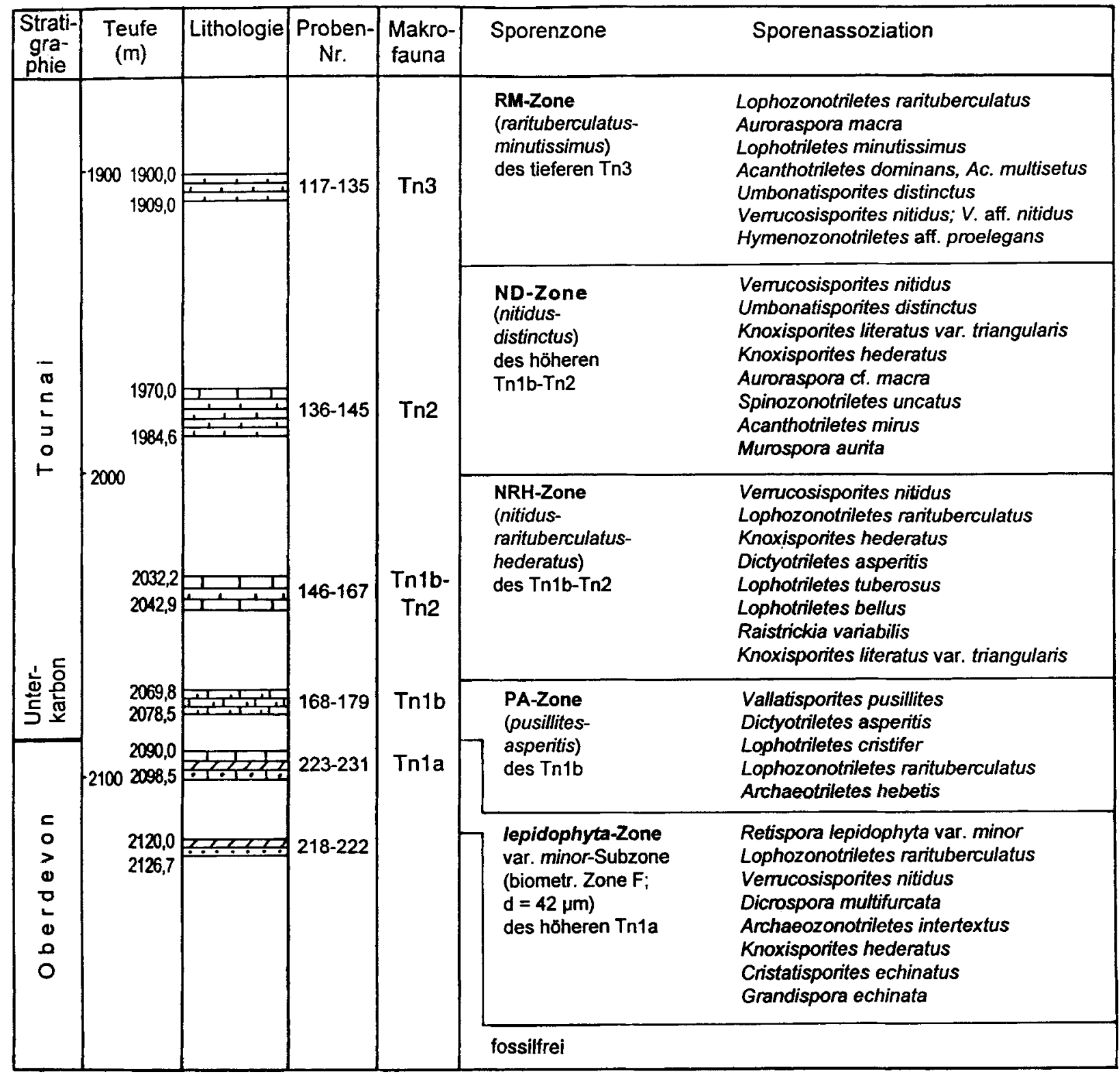

Tabelle 1. Sporen an der Devon-Karbon-Grenze und aus dem Tournai der Bohrung Neuenkirchen 2/73

la ist in beiden Bohrungen an die makrofaunistisch angenommene V1a-Basis gebunden und entspricht der Rolle der überregional definierten Pu-Zone (sensu lato) in Bezug auf die Tournai/ Visé-Grenze. Die Obergrenze der Pu-Zone ist in beiden Bohrungen nicht erfassbar (fehlende Kernanteile, prä-Westfal-Erosion am Top der Unterkarbon-Profile).

Der überregionale Umfang der Pu-Zone (sensu lato) wird nachfolgend der regionalen Gliederung auf Rügen gegenübergestellt. Die Einschaltung des Gloeocapsamorpha-Horizontes innerhalb des V1a ermöglicht die diffizilere Gliederung (Tab. 4).

Überregionale sporenstratigraphische Vergleiche (Tab. 5) wurden seinerzeit für das Tournai (Burmann 1975) bevorzugt mit der Pripjat-Senke vorgenommen, in der Kedo (1957a-c, 1963, Ke- do \& Golubzow 1971) zahlreiche Profile bearbeitet hatte. Neuere Untersuchungen hat Avchimovitch (1993) für die Pripjat-Senke vorgenommen mit Einbeziehung überregionaler Einbindungen (Avchimovitch et al. 1993, Avchimovitch \& Turnau 1994), auf die hier nur verwiesen wird. Auch zur Russischen Tafel (Byvsheva et al. 1984, Byvsheva \& Umnova 1993) bestehen gute Vergleichsmöglichkeiten. Ein detaillierter Vergleich würde aber einen umfangreichen taxonomischen Aufwand bedeuten (Clayton 1996, Avchimovitch 1993). Ein Teil der in der Literatur als Indexfossilien geltenden Arten ist in manchen Regionen schwer nachweisbar, andere wie Dictyotriletes asperitis, die sich auf Rügen als gut erkennbare Komponenten mit begrenzter Reichweite darstellen und auch in der Pripjat-Senke belegt sind, 


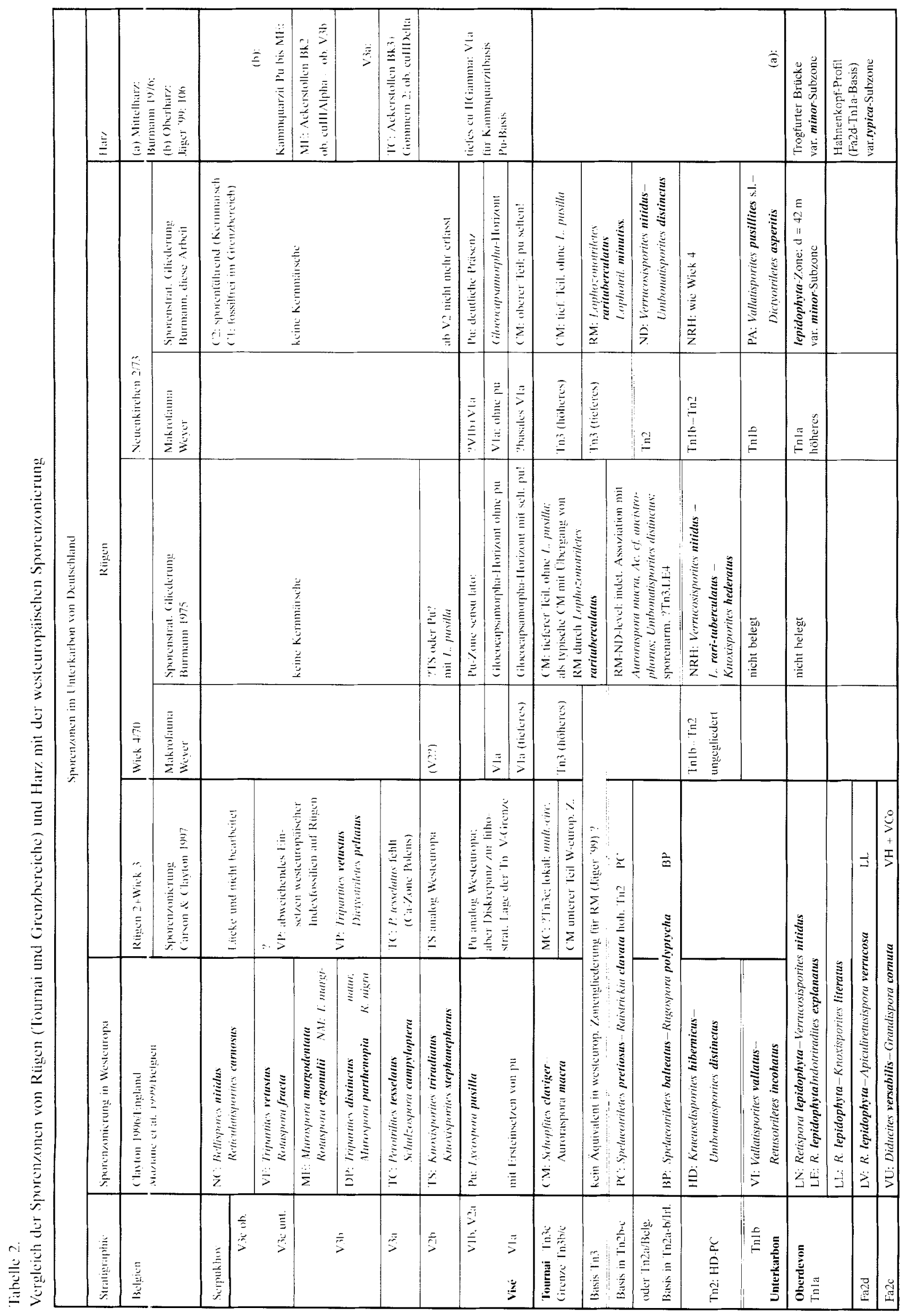


Tabelle 3

Sporenzonen im Unterkarbon der Bohrung Neuenkirchen 2/1973

(mit Vergleichsangaben aus den Bohrungen Wiek 4/1970 und Sagard 1/1970)

\begin{tabular}{|c|c|c|c|c|}
\hline Stratigraphie & Kermmarsch (m) & Proben-Nr. & Sporenzone & Bohrungsvergleich \\
\hline Oberkarbon & $\begin{array}{l}1420,8 \text { - } \\
(1425 \mathrm{~m} \text { Grenze })\end{array}$ & & sporenführend & \\
\hline \multirow[t]{2}{*}{ Unterkarbon } & $\begin{array}{l}-1428,2 \mathrm{~m} \\
1428,2-1433,1\end{array}$ & $\begin{array}{l}180-185 \\
186-187\end{array}$ & fossilleer & \\
\hline & & & Kernlücke & $\begin{array}{l}\text { Wiek 4: TS oder Pu (V2?) } \\
1342,2-1351,3 \mathrm{~m} \\
1460,6-1469.6 \mathrm{~m}\end{array}$ \\
\hline Vlb & $1517,0-1526,6$ & $188-214$ & $\begin{array}{l}\text { Pu-Zone mit } \\
\text { Lycospora pusilla }\end{array}$ & $\begin{array}{l}\text { Wiek 4: } \\
\text { Kernlücke }\end{array}$ \\
\hline V1a & $\begin{array}{l}1585,0-1602,5 \\
1602,5-1620,5 \\
\end{array}$ & $\begin{array}{l}215-217 \\
1-16\end{array}$ & $\begin{array}{l}\text { Pu-Zone } \\
\text { Pu-Zone }\end{array}$ & \\
\hline V1a & $\begin{array}{l}1673,0-1684,6 \\
1684,6-1702,6\end{array}$ & $\begin{array}{l}17-39 \\
40-61\end{array}$ & $\begin{array}{l}\mathrm{CM}=\text { Pu s.l. bzw. Gloeocapsamor- } \\
\text { pha-Horizont mit Tornacia sarjeanti, } \\
\text { Rugospora, Gloeocapsmorpha prisca } \\
\text { dto. } \\
\text { ohne L. pusilla }\end{array}$ & $\begin{array}{l}\text { Gloeocapsamorpha-Horizont } \\
\text { Wiek 4: V1a; mit Lyc: pusilla } \\
\text { 1732,0-1739.8 m } \\
\text { Wiek 4: (Tn/V:Vla) } \\
\text { 1844,4-1853,4 m }\end{array}$ \\
\hline Vla & $1780,0-1791,3$ & $62-86$ & $\begin{array}{l}\text { CM-Zone (höherer Teil) = Pu s.l. } \\
\text { mit seltenen L. pusilla }\end{array}$ & $\begin{array}{l}\text { Wiek 4: } \\
\text { Kernlücke }\end{array}$ \\
\hline Tournai & & & $\begin{array}{l}\text { CM-Zone (tieferer Teil) } \\
\text { ohne L. pusilla }\end{array}$ & Wiek 4: \\
\hline $\begin{array}{l}\text { Tn3 } \\
\text { (höheres) }\end{array}$ & $\begin{array}{l}1810,0-1818,8 \\
1818,8-1827,8\end{array}$ & $\begin{array}{l}87-105 \\
101-116\end{array}$ & $\begin{array}{l}\text { CM Schopfites claviger- } \\
\text { CM Auroraspora macra }\end{array}$ & Kernlücke \\
\hline \multirow{3}{*}{$\begin{array}{l}\operatorname{Tn} 3 \\
\text { (ticferes) }\end{array}$} & & & & $\begin{array}{l}\text { Wiek 4: hangende RM/CM } \\
1978,7-1987,7\end{array}$ \\
\hline & $1900.0-1909.0$ & $117-135$ & $\begin{array}{l}\text { RM-Zone } \\
\text { mit } \\
\text { Lophozonotriletes } \\
\text { rarituberculatus } \\
\text { Lophotriletes } \\
\text { minutissimus }\end{array}$ & $\begin{array}{l}\text { Wiek 4: RM vermutl.zwischen } \\
\text { 1978,7-1987,7 m Tn3 } \\
2101,1-2109,3 \mathrm{~m} \mathrm{Tn} 3 \\
\text { Sagard 1: RM } \\
2385,0-2389,9 \mathrm{~m} \\
\text { (tieferes Tn3) }\end{array}$ \\
\hline & & & & $\begin{array}{l}\text { Wiek 4: indet. ?bas. RM/ND } \\
2101,1-2109,3 \mathrm{~m} \mathrm{Tn3}\end{array}$ \\
\hline $\operatorname{Tn} 2$ & $1970,0-1984,6$ & $136-145$ & $\begin{array}{l}\text { ND-Zone mit } \\
\text { Verrucosisporites nitidus - } \\
\text { Umbonatisporites distinctus }\end{array}$ & $\begin{array}{l}\text { Wiek 4: } \\
\text { Kernlücke }\end{array}$ \\
\hline $\operatorname{Tn} 1 b-\operatorname{Tn} 2$ & $2032,2-2042,9$ & $146-167$ & $\begin{array}{l}\text { NRH-Zone mit } \\
\text { Verrucosisporites nitidus - } \\
\text { Lophozonotriletes raritubercu- } \\
\text { latus - Knoxisporites hederatus }\end{array}$ & $\begin{array}{l}\text { Wiek 4: NRH Tn1b-Tn2 } \\
2228,2-2237,2 \mathrm{~m}\end{array}$ \\
\hline $\operatorname{Tn} 1 b$ & $2069,8-2078.5$ & $168-179$ & $\begin{array}{l}\text { PA-Zone mit } \\
\text { Vallatisporites pusillites- } \\
\text { Dictyotriletes asperitis }\end{array}$ & \\
\hline \multirow[t]{2}{*}{$\begin{array}{l}\text { Tn1a } \\
\text { (höheres) }\end{array}$} & $2090,0-2098,5$ & $223-231$ & $\begin{array}{l}\text { Le-Zone mit } \\
\text { Retispora lepidophyta } \\
\text { var. minor-Subzone } \\
\text { (biometr. Zone } F=42 \mathrm{~m} \text { ) }\end{array}$ & \\
\hline & $2120,0-2126.7$ & $213-222$ & fossilfrei & \\
\hline
\end{tabular}

sind in der Literatur stratigraphisch kaum gewichtet. Der in Burmann (1975) geführte Vergleich mit dem Tournai der Pripjat-Senke anhand der von Kedo (1957a-c, 1963, 1971) vorgelegten Daten ist nach wie vor gültig (Tab. 5).
Als sporenstratigraphisches Standard-Vergleichsgebiet für den Devon/Karbon-Grenzbereich gilt das Ardenno-Rhenische Becken (Paproth \& Streel 1971, Streel in Becker et al. 1974, Higgs \& Streel 1984, Streel et al. 1987. 
Tabelle 4

\begin{tabular}{|c|c|c|}
\hline & $\begin{array}{l}\text { Regionale Gliederung der Pu-Zone in den } \\
\text { Bohrungen Wiek } 4 \text { und Neuenkirchen } 2\end{array}$ & $\begin{array}{l}\text { Überregionale Anwendung } \\
\text { der Pu-Zone (sensu lato) }\end{array}$ \\
\hline \multirow{3}{*}{ Hangendes: } & \multirow{3}{*}{$\begin{array}{l}\text { Obergrenze der Pu-Zone } \\
\text { profilbedingt ab V2a nicht erfasst }\end{array}$} & $\mathrm{TC}=$ Ca-Zone Polens (V3a) \\
\hline & & $\begin{array}{l}\text { In Polen auch V2b } \\
\text { für Pu-Zone. } \\
\text { TS-Zone W-Europa, Rügen }\end{array}$ \\
\hline & & $\mathrm{V} 2 \mathrm{a}$ \\
\hline \multirow[t]{2}{*}{ V1b+höh.V1a } & $\mathrm{Pu}$ (pusilla)-Zone mit Lycospora pusilla & \\
\hline & Gloeocapsamorpha-Horizont & $\begin{array}{l}\text { pusilla-Zone (sensu lato) } \\
\text { Westeuropas }\end{array}$ \\
\hline Vla & $\begin{array}{l}\text { in der Wiek } 4 \text { mit seltenen Lycospora pusilla } \\
\text { in der Neuenkirchen } 2 \text { ohne Lycospora pusilla }\end{array}$ & \\
\hline Vla (tieferes) & $\begin{array}{l}\text { oberer Teil der CM (claviger-macra-Zone) } \\
\text { mit sehr seltenen Lycospora pusilla = Pu s.l. }\end{array}$ & $\begin{array}{l}\text { Einsetzen der } \mathrm{Pu} \text {-Zone } \\
\text { im basalen Visé } \\
\text { V1a }\end{array}$ \\
\hline $\begin{array}{l}\text { Liegendes: } \\
\text { Tn3 (höheres) }\end{array}$ & $\begin{array}{l}\text { unterer Teil der CM-Zone } \\
\text { ohne Lycospora pusilla }\end{array}$ & Tournai \\
\hline
\end{tabular}

Tabelle 5

Korrelation der Sporenzonen aus der Bohrung Neuenkirchen $2 / 73$ mit den Sporenzonen in der Pripjat-Senke, Bjelorussia (Kedo 1957. 1963. 1971. 1974)

\begin{tabular}{|c|c|c|c|}
\hline Stratigraphie & Kernmarsch (m) & Sporenassoziation von Rügen & Sporenkomplex der Pripjat-Senke \\
\hline $\mathrm{V} 1 \mathrm{~b}$ & $1517.0-1526.9$ & Pu-Zone & \multirow{5}{*}{$\begin{array}{l}\text { br2-Komplex der } \\
\text { mittleren } \\
\text { Kaolin-Schichten }\end{array}$} \\
\hline Vla & $1585.0-1602.5$ & $\mathrm{Pu}$-Zone & \\
\hline V1a & $1602.5-1620,5$ & $\mathrm{Pu}$-Zone & \\
\hline & $1673,0-1684.6$ & Gloeocapsamorpha- & \\
\hline V1a & $1684.6-1702.6$ & Maximum & \\
\hline Vla? & $1780.0-1791.3$ & $\begin{array}{l}\text { Pu-Übergang: } \\
\text { höhere CM-Zone }\end{array}$ & \multirow{3}{*}{$\begin{array}{l}\text { br } 1 \text {-Komplex der } \\
\text { unteren Kaolin-Scht. }\end{array}$} \\
\hline $\operatorname{Tn} 3$ & $1810.0-1818.8$ & & \\
\hline & $1818.8-1827.8$ & CM-Zone: höheres Tn3 & \\
\hline $\operatorname{Tn} 3$ & $1900.0-1909.0$ & RM-Zone: tieferes $\operatorname{Tn} 3$ & $\begin{array}{l}\text { kis2-Komplex der } \\
\text { oberen Kiselow-Scht. }\end{array}$ \\
\hline $\operatorname{Tn} 2$ & $1970.0-1984.6$ & ND-Zone & $\begin{array}{l}\text { ?kis1-Komplex Kiselow } \\
\text { ?ch1-3 Tscherepet }\end{array}$ \\
\hline $\operatorname{Tn} 1 b-\operatorname{Tn} 2$ & $2032.2-2042.9$ & NRH-Zone & $? \mathrm{ml}$-3 Malev \\
\hline $\operatorname{Tn} 1 b-\operatorname{Tn} 2$ & $2069.8-2078,5$ & PA-Zone & $\begin{array}{l}\text { ml1-Komplex (pusillites-Z.) } \\
\text { der unt. Malev-Scht. }\end{array}$ \\
\hline Tnla & $2090.0-2098.5$ & $\begin{array}{l}\text { lepidophyta var. minor- } \\
\text { Subzone: biometr. Zone F: }\end{array}$ & $\begin{array}{l}\text { IIIB-Komplex der } \\
\text { Oser-Chovan-Schichten }\end{array}$ \\
\hline
\end{tabular}

Maziane et al. 1999). Für die auf Rügen (Bohrung Neuenkirchen 2) und im Mittelharz (Trogfurter Profil; Bielstein; Hahnenkopf - WSW Elbingerode) bisher nachgewiesenen Vorkommen der lepidophyta-Zone aus dem östlichen Teil Deutschlands hat sich die von Streel entwikkelte Subzone ngliederung auf der Basis der zum Hangenden entretenden Größenabnahme von Retispora lepilophyta als die praktikablere Variante der Zcnenzuordnung bei Einzelvorkommen erwiesen.

\section{Vergleich der Sporenzonen aus den Bohrungen Wiek 4/1970 und Neuenkirchen 2/1973}

\section{lepidophyta-Zone}

Die lepidophyta-Zone des höchsten Famenne ist auf Rügen nur in der Bohrung Neuenkirchen 2 belegt (im Profilverband mit der tiefsten unterkarbonischen pusillites-asperitis- bzw. PA-Sporenzone, lediglich getrennt durch eine Meißelstrecke von $11.5 \mathrm{~m}$ ). Das Vorkommen gehört zur biometrischen Zone $\mathrm{F}(\mathrm{d}=42 \mu \mathrm{m})$ der lepidophyta var. minor-Subzone (höheres Tn1a) und ist vergleich- 
bar mit der biometrischen Zone $\mathrm{F}$ des höheren Tn1a im Ardenno-Rhenischen Becken und dem IIIB-Sporenkomplex der Oser-Chovan-Schichten in der Pripjat-Senke. In anderen Rügen-Bohrungen ist das Niveau nach Bohrloch-Geophysik ebenfalls präsent, wurde aber nicht durch Kernstrecken erfasst.

\section{PA-Zone}

Die PA-Zone (Vallatisporites pusillites - Dictyotriletes asperitis) ist in der Bohrung Neuenkirchen 2 makrofaunistisch in das Tn1b eingestuft. In der Bohrung Wiek 4 ist diese Strecke nicht gekernt worden. Die Verhältnisse auf Rügen entsprechen weitgehend der pusillites-Zone in den unteren Malev-Schichten ( $\mathrm{ml}_{1}$-Sporenkomplex; ebenfalls mit beiden Indexfossilien) der Pripjat-Senke (Kedo 1963, 1966, 1971, Burmann 1975: 883).

Unter der älteren Bezeichnung Hymenozonotriletes pusillites ist das Vorkommen der Art auch in der lepidophyta var. typica-Subzone des Harzes dokumentiert (Burmann 1976: 809) in Analogie zu den Verhältnissen im Ardenno-Rhenischen Becken. In der Gliederung von Neves et al. (1972) ist Vallatisporites pusillites ein Indexfossil der PL-Zone zusammen mit Retispora lepidophyta im Tn1a. Auf Rügen (Tn1b) und in den unteren Malev-Schichten der Pripjat-Senke ist Vallatisporites pusillites (sensu lato) aber noch oberhalb des Etroeungt verbreitet, eben in der PA- bzw. pusillites-Zone.

Die basale Sporenzone der westeuropäischen Gliederung (Clayton 1996: 592) stellt die VI-Zone dar (Vallatisporites verrucosus - Retusotriletes incohatus), die dem Tn1b entspricht und damit die gleiche Position einnimmt wie die PA-Zone.

\section{NRH-Zone}

Die NRH-Zone (Verrucosisporites nitidus - Lophozonotriletes rarituberculatus - Knoxisporites hederatus) im Tn1b-Tn2 ist in beiden Bohrungen, der Neuenkirchen 2 und Wiek 4, erfasst. Es zeichnet sich ein oberes Häufigkeitsoptimum von Knoxisporites hederatus und ein unteres Häufigkeitsoptimum mit Dictyotriletes asperitis ab. Weitere Arbeiten sind jedoch in diesem Abschnitt zur Ermittlung zusätzlicher zonenspezifischer Komponenten erforderlich (Dictyotriletes asperitis auch oberhalb der pusillites-asperitis-Zone, Lophotriletes tuberosus, Knoxisporites literatus var. triangularis).

\section{ND-Zone}

Die ND-Zone (Verrucosisporites nitidus - Umbonatisporites distinctus) wurde bisher nur in der Bohrung Neuenkirchen 2 ausgegliedert mit makrofaunistischer Eichung als Tn2 zwischen der NRH-Zone im Liegenden und der RM-Zone im Hangenden. Sie ist in der Bohrung Wiek 4 (Burmann 1975: 883) nicht ausgehalten zwischen der NRH-Zone (Tn1b-Tn2 ungegliedert) und der sporenarmen, möglicherweise basalen RMZone (tieferes Tn3); offenbar entfällt sie dort auf die Meißelstrecken zwischen den nur sporadisch vorliegenden Kernmärschen (Tab. 3). Die Übersichtsdarstellung für Rügen in Burmann (1975: 881) als ND-Subzone basierte bereits auf dem Nachweis in der Bohrung Neuenkirchen 2.

Nach der Beschreibung der BP-Zone (Spelaeotriletes balteatus - Rugospora polyptycha) bei Carson \& Clayton (1997: 220) dürfte diese der ohne Bohrungsbenennung publizierten (Burmann 1975: 881) ND-Zone des Tn2 entsprechen, die hier (Tab. 1) nunmehr fundortsbezogen der Bohrung Neuenkirchen 2 zugeordnet wurde. Nach Higgs et al. (1988a; in Carson \& Clayton 1997: 222) entspricht die BP-Zone dem Mitteltournai Tn2a-Tn2b; nach Higgs et al. (1992; in Carson \& Clayton 1997: 222) soll die BP-Zone in Belgien dem Tn2a (Untere Siphonodella crenulata-Conodontenzone) entsprechen.

Von Higgs et al. (1988a in Carson \& Clayton 1997: 222) wurde der obere Teil der ND-Zone (Burmann 1975) als Äquivalent zur westeuropäischen BP-Zone und zum unteren Teil der PCZone angesehen. Dem kann insofern zugestimmt werden, als man hier bei der überregionalen Korrelation wohl an den Grenzen der stratigraphischen Auflösung steht (Tab. 2).

\section{RM-Zone}

Die Assoziation der RM-Zone enthält neben Kleinsporen häufig Lophozonotriletes rarituberculatus und seltene Verrucosisporites nitidus sowie häufig Auroraspora macra und auch Umbonatisporites distinctus.

Die RM-Zone (Lophozonotriletes rarituberculatus - Lophotriletes minutissimus) wurde seinerzeit erstmals auf Rügen in der Bohrung Sagard 1/1970 erkannt, konnte aber zunächst makrofaunistisch nur zwischen Tn1b-Tn2 und V1b eingegrenzt werden und wurde sporenstratigraphisch dem Übergangsbereich $\operatorname{Tn} 2 / \mathrm{Tn} 3$ zugeordnet. Die RM-Zone konnte dann in der Bohrung Neuen- 
kirchen 2 (Tah. 1) makrofaunistisch geeicht werden (Tn3: tieferes Tn3 sporenstratigraphisch ableitbar). Im P ofil der Bohrung Wiek 4 sind die Verhältnisse weniger klar: vermutlich ist nur der hangende Teil der RM-Zone (noch mit L. rarituberculatus) an Übergang zur (M-Zone gekernt worden und die eigentliche RM-Zone entfällt auf eine Kernlücke im Liegenden.

Dic stratigraphische Position kann durch die Situation in der Bohrung Wiek 4 (mit 2 Kernstrecken im $\operatorname{Tn} 3$ ) wie folgt eingegrenzt werden: der obere $\operatorname{Tr} 3$-Kernmarsch (1978.7-1987.7 m) bildet wahrsch einlich die äußerste Hangendgrenze der RM-Zone bzw. Basis der CM-Zone. und der tiefere Tn3-K.ernmarsch bei 2101.1-2109.3 m (Basis der lithologischen Einheit $4=$ Tn3-Basis von Rügen) zuigt noch nicht dic typische Ausbildung der RM-Zone (?faziesbedingt: geringere Sporenführungr): die eigentliche RM-Zone fällt demnach in cie Meißelstrecke zwischen beiden Tn3-Kernmärschen.

Die RM-Zone entspricht dem tieferen Teil des Tn3, das sich sporenstratigraphisch unterteilen lässt (höheres $\mathrm{Tn} 3=\mathrm{CM}$-Zone). Die RM-Zone von Rügen ist aufgrund des genannten Kriteriums gut mit dem sis-Komplex der oberen KiselowSchichten in der Pripjat-Senke zu korrelieren.

\section{CM-Zone, tieferer Teil}

Die CM-Zon: (Schopfites claviger - Auroraspora macra) ist für Rügen in den Bohrungen Wick 4 und Veuenkirchen 2 in einen tieferen Teil ohne seltene Lycospora pusilla (makrofaunistisch $\operatorname{Tn} 3$, sporenstratigraphisch höheres $\operatorname{Tn} 3$ ) und einen hölıeren Teil (makrofaunistisch tieferes V1a) mit sehr seltenen Lycospora pusilla (unterhalb und innerhalb des Gloeocapsamorpha-Horizontes) untergliedert. Für das Tn3. das auf Rügen sonst biostratigraphisch nicht weiter untergliederbar ist. ist sporenstratigraphisch eine Gliederung in tieferes Tn3 (RM-Zone) und höheres Tn3 (CM-Zone ohne L. pusilla) möglich. Unsicherheiten rühren aus den Kernmarschlücken her, sodass eine wejtere Kontrollmöglichkeit wünschen iwert wäre.

Der höhere Teil der CM-Zone entspricht dadurch bereits dem basalen Abschnitt der überregionalen Pu Zone (sensu lato) - das Abgrenzungskriterium ist jedoch schwer zu erfassen. weil es nicht immer gelingt. den Nachweis von Lycospora puilla zu führen: es erscheint fraglich ob dieses Niveau bereits der Pu-Zone in der Pripjat-Senke (br-Komplex der mittleren Kao-
lin-Schichten) entspricht. Deshalb ist es für dic lokale Glicderung akzeptabler, die Assoziation solange in der CM-Zone zu belassen, bis der eindeutige Nachweis von Lycospora pusilla geführt ist. und zwischenzeitig das lokale Hilfskriterium von Gloeocapsamorpha für die Abgrenzung einzusetzen.

Die von Carson \& Clayton (1997: 223) in den Bohrungen Wiek 3 und Rügen 2 zwischen der CM-Zone und der Pu-Zone im höheren Tournai von Rügen abgetrennte MC-Zone (Gorgonispora multiplicabilis - Convolutispora circumvallata) soll dem oberen Teil der CM-Zone Westeuropas entsprechen und außer dem Einsetzen von G. multiplicabilis ansonsten eine der CMZone vergleichbare Sporenassoziation ausweisen (Jäger 1999: 89). Durch das Fehlen von Lycospora pusilla müsste sie nach unserem Verständnis in den .,tieferen Teil der CM-Zone“, d. h. in den Hauptteil der CM-Zone von Rügen eingehen (Tab. 1).

Für uns ist die Relevanz einer solchen lokalen Zone nicht erkennbar, und auch Carson selbst sieht sie als eine lokale Erscheinung auf Rügen an. da die Indexart Gorgonispora multiplicabilis in Polen bereits früher erscheint. Offen bleibt allerdings die Frage, wie die stratigraphische Aussage der MC-Zone als höheres Tn3 abgeleitet wurde. wenn in den Bohrungen Rügen 2 und Wiek 3. in denen sie zwischen Pu-Zone und CMZone etabliert wurde, eine Diskrepanz zwischen der Sporenstratigraphie und der lithostratigraphisch/makrofaunistischen Gliederung in Bezug auf die Lage der Tournai/Visé-Grenze besteht (Carson \& Clayton 1997: 221).

\section{CM-Zone, höherer Teil (= Pu-Zone sensu lato $)$}

Der höhere Teil der CM-Zone, der bereits dem Vla entspricht, weist sporadisch Lycospora pusilla auf, er gehört also zur Pu-Zone sensu lato. Dazu gehört im V1a ein basales Niveau unterhalb des Gloeocapsamorpha-Horizontes (nur in der Neucnkirchen 2 erfasst) sowie der Gloeocapsamorpha-Horizont selbst, der eventuell auch aus mehreren Horizonten besteht.

Für die Bohrung Neuenkirchen 2 wurde das Niveau mit den häufigen Vertretern von Gloeocapsamorpha prisca als selbstständiger Horizont ausgeschieden (makrofaunistisch als V1a datiert. bisher ohne Vergleichsmöglichkeiten außerhalb Rügens). Sporenstratigraphisch liegt er zwischen dem oberen Teil der CM-Zone (mit sehr seltenen Lycospora pusilla) im Liegenden und der 
Pu-Zone (im Sinne einer erkennbaren häufigeren Verbreitung von Lycospora pusilla, höheres V1a und V1b?) im Hangenden. In der Bohrung Neuenkirchen 2 wurden im GloeocapsamorphaHorizont keine Lycospora pusilla gefunden.

In der Bohrung Wiek 4 entspricht das Häufigkeitsoptimum von Gloeocapsamorpha prisca (Burmann 1975: 883) dem makrofaunistisch ebenfalls als V1a datierten oberen Teil der CMZone. Lycospora pusilla ist hier, wenn auch sehr selten, im Gloeocapsamorpha-Horizont nachgewiesen $(1844,4-1853,3 \mathrm{~m}$ und 1732,0-1739,8 m), wodurch sich dieser Abschnitt vom unteren Teil der CM-Zone des höheren Tn3 absetzt. Es besteht also ein Unterschied zwischen den Bohrungen Wiek 4 und Neuenkirchen 2 in Bezug auf die Nachweismöglichkeit sehr sporadisch auftretender Lycospora pusilla im GloeocapsamorphaHorizont.

Es bleibt hier mehr eine Definitionssache, wie der Umfang der Pu-Zone gefasst wird, um auch regional damit arbeiten zu können. Die Übertragung des Umfangs der Pu-Zone der westeuropäischen Gliederung mit dem seltenen Ersteinsetzen von Lycospora pusilla als Zonenbasis kann zwar für die überregionale Korrelation von Nutzen sein (Pu-Zone sensu lato), schränkt aber die regionalen Gliederungs- und Korrelationsmöglichkeiten ein. Ihr Ersteinsetzen ist zu selten erfassbar, als dass auf ein regionales Hilfskriterium wie den Gloeocapsamorpha-Horizont verzichtet werden könnte.

Der Umfang der Pu-Zone im Sinne der westeuropäischen Gliederung wird deshalb auf Rügen gegliedert in den oberen Teil der CM-Zone, in der seltene Lycospora pusilla nachgewiesen sind (basales V1a), in den darüber folgenden Gloeocapsamorpha-Horizont (V1a, ? tieferes bis mittleres), und die eigentliche Pu-Zone mit zweifelsfreier häufigerer Präsenz von Lycospora pusilla (ab dem höheren V1a in der Bohrung Neuenkirchen 2).

Letztere entspricht in der Pripjat-Senke dem $\mathrm{br}_{2}$-Komplex der mittleren Bobrikow-Schichten unterhalb der produktiven Serie der eigentlichen Bobrikow-Schichten des Moskauer Beckens. Sporenstratigraphisch scheint jedoch überregional die Basis der Pu-Zone in Großbritannien und Rügen gleichermaßen im Bereich der Tournai/Visé-Grenze vorzuliegen, wie es die makrofaunistische Eichung des oberen Teils der CMZone der Bohrungen Wiek 4 und Neuenkirchen 2 als tieferes V1a bestätigt.

Carson \& Clayton (1997: 222) argumentieren zur Definition der Pu-Zone, dass „.... in the Rü- gen Dinantian succession this taxon is never common" und man deshalb keine Modifizierung zwischen Einsetzen von ,significant numbers" (im höheren Bereich) und "first appearence" von seltenen Formen von Lycospora pusilla im Basisbereich vornehmen könne (Bezug zu Burmann 1975: 883 über eine modifizierte Verwendung des Begriffes Pu-Zone für regionale Aspekte). Auch wenn die Definition von Neves et al. (1972) zum (seltenen) Ersteinsetzen als Basis der Pu-Zone für die überregionale Korrelation akzeptabel erscheint, ist der Einsatz zusätzlicher Kriterien für die lokale Gliederung erforderlich. Für die Bohrungen Wiek 4 und Neuenkirchen 2 wurde eine solche Differenzierung im Niveau der Pu-Zone (sensu lato, z. B. gemäß Neves et al. 1972) versucht.

\section{Zur Tournai-Visé-Grenze}

Zur Fassung der Pu-Zone (sensu lato) auf der Basis der Definition von Neves et al. (1972: Ersteinsetzen von Lycospora pusilla) für überregionale Korrelationszwecke besteht überwiegend Übereinstimmung, weitgehend auch über die Position dieses Niveaus im Bereich der Tournai/ Visé-Grenze. Im Einzelfall besteht aber oft das Nachweisproblem des wirklich ersten Einsetzens der oft seltenen Indexart. Falls dies nicht gelingt. kann die Tournai/Visé-Grenze kaum erfasst werden; deshalb sind Hilfskriterien wie das des Rügener Algenhorizontes mit Gloeocapsamorpha von Bedeutung.

Für die Bohrungen Wiek 4 und Neuenkirchen 2 (Tab. 2) ist die Position des Gloeocapsamorpha-Horizontes makrofaunistisch im Vla angesiedelt und etwa gleichaltrig. Da im Liegenden (oberer Teil der CM-Zone bzw. Pu-Zone sensu lato) in beiden Bohrungen jeweils auch Lycospora pusilla sehr selten auftritt, gehört der Gloeocapsamorpha-Horizont in die Pu-Zone. Lithostratigraphisch befindet er sich im Niveau der Lithoeinheiten (sensu Hoffmann et al. 1975) Top-5 bis Top-11 (Bohrung Wiek 4, Nachweis bei Teufe $1850 \mathrm{~m}$ und $1735 \mathrm{~m}$ ), d. h. fast im gesamten V1a.

Carson \& Clayton (1997: 221) vermerken eine Diskrepanz ihrer sporenstratigraphischen Ergebnisse zur Lage der Tournai/Visé-Grenze in den Bohrungen Rügen 2 und Wiek 3 mit der Grenzziehung, die von Hoffmann et al. (1975) vorgenommen wurde: „The Tournaisian/Viséan boundary of Hoffmann et al. at the base of Unit 5 in the Rügen succession is considerably lower than 
that suggested by the miospore evidence. at the base of Unit ' 3 or the top of Unit 12". Dieser Widerspruch i'st ohne Diskussion möglicher Ursachen nicht 'u akzeptieren. Am Beispiel der Bohrungen Neuenkirchen 2 und Wiek 4 ist ersichtlich, dass eine solche Diskrepanz nicht existiert - die Datierungen nach Makrofauna und Sporen stimmen durchaus überein.

Auch Weye' (2001: 62) und Hoffmann et al. (2002) betonell. dass cine so weitgehende palynologische Verschiebung der Tournai/Visé-Grenze von der Eiasis der Litho-Einheit 5 in den Grenzbereich von Litho-Einheit 12/13 (real Arundium-Basis) bei Carson \& Clayton (1997: 221, 222) ".nic it akzeptabel" ist und dass in der Bohrung Wiek 3 das ..... wirklich erste Einsetzen der auf Rügen offenbar seltenen Leitart" Lycospora pusilla iiberhaupt nicht erfasst wurde: sie verweisen auf den bereits eindeutigen Nachweis der Art im V1a von Rügen durch Burmann (1975: Tab. 2). was bei Carson \& Clayton (1997) unberücksichtigt blieb. Der Grenzbereich der Litho-Einheiten 5 und 6 im Kernmarsch 1844,4-1853,4 m der Bohrung Wiek 4 enthält bereits den Gloeocapsamorpha-Horizont mit Lycospora pusilla. Bei Carson \& Clayton (1997) ist diese Algenkomponente für die Bohrungen Rügen 2 und Wiek 3 leider nicht erwähnt. sie ist aber z. B. auch aus der Wiek 101 bekannt: in der Sporenzor engliederung von England ist die Komponente richt benannt.

Es wäre Spskulation. sich über die Ursachen der angeblich€n Diskrepanz zur Lage der Tournai/Visé-Grenze bei Carson \& Clayton (1997) zu äußern. da die Autoren nicht näher auf ihre methodischen Vergleichsgrundlagen eingegangen sind. Es fällt auf. dass die von ihnen aufgestellte neue MC-Zon: (die nur lokale Bedeutung haben soll, da ihr Indexfossil in Polen bereits mit der CM-Zone einsetzt) in der Bohrung Wiek 3 ein Mehrfaches an Mächtigkeit aufweist im Vergleich zur CV-Zone bei ansonsten gleichen Komponenten. Auch sind mögliche weitere Hilfskriterien, wie das Auftreten von Densosporites oder weiterer, durchaus vorhandener Algenkomponenten, nicht in die Betrachtung einbezogen worden, um die Nachweissicherheit zum tatsächlichen Einsetzen von Lycospora erhöhen zu können.

Jäger (1999: 83) betont als eine der Abweichungen zwischen Rügen und der westeuropäischen Zonierung die unterschiedliche Verbreitung von Densosporites ( $D$. annulatus, $D$. intermedius, $D$ spinifer $u$. a.). In Rügen und der Hörre-Gommern-Quarzitzone setzen sie ..... ein- deutig vor Lvcospora pusilla, der Indexspore für die Visé-Basis, im obersten Tournai (CM-Zone) ein... In Westeuropa setzen sie dagegen deutlich nach Lycospora pusilla im mittleren Visé (TCZone) ein". Als typisch für die polnische Mikroflora weist Jäger (1999: 83) auf eine Gemcinsamkeit zu Mitteleuropa hin, das „... extrem seltene Auftreten oder Fehlen von Lycospora im untersten Visé...": als deutsches Vergleichsbeispiel führt er cin Profil vom Kammquarzit aus dem Ackerstollen (Bk4) im Oberharz an. Diese Tatsache wird als Ursache für die lange Zeit von Westeuropa abweichende Zonicrung des tiefen Visé in Polen angegeben. In der Bohrung Neuenkirchen 2 tritt Densosporites sp. erstmals in der CM-Zone des höheren Tn3 auf, also noch vor der seltenen Lycospora pusilla, ist aber ebenfalls keine auffällige Komponente. In der Bohrung Wiek 4 wurde Densosporites sp. zusammen mit L. pusilla im Gloeocapsamorpha-Horizont im Vla beobachtet.

\section{Ergebnisse}

1. Im Vergleich zu Burmann (1975) können für das verallgemeinerte Sporenzonierungsschema des Tournai und seiner Grenzbereiche von Rügen nunmehr die konkreten Daten aus den Bohrungen Neuenkirchen 2 und Wiek 4 vorgelegt werden. was aus aktuellen Gegebenheiten in den siebziger Jahren nicht möglich war. Trotz beträchtlicher Kernlücken (Tabelle 1) erweist sich das Profil der Bohrung Neuenkirchen 2 als gecignet. zwischen der lepidophyta var. minor-Subzone (höheres Tn1a) im Liegenden und der pusilla-Zone (ab V1a) im Hangenden mehrere weitere Sporenzonen zu unterscheiden, die auch makrofaunistisch eingebunden sind (Tabelle 2):

- PA-Zone (pusillites-asperitis) im Tn1b (wie die lepidophyta-Zone sporenstratigraphisch nur in der Bohrung Neuenkirchen 2 erfasst);

- NRH-Zone (nitidus-rarituberculatus-hederatus) aus dem Tn1b-Tn2 (die Sporenassoziation wurde in Burmann 1975 aus der Bohrung Wiek 4 abgebildet und liegt im gleichen stratigraphischen Niveau aus der Neuenkirchen 2 vor):

- ND-Zone (nitidus-distinctus) aus dem Tn2 (das Niveau ist nur in der Bohrung Neuenkirchen 2 sicher erfasst);

- RM-Zone (rarituberculatus-minutissimus) aus dem tieferen Tn3, bereits mit Kleinsporen der $\mathrm{CM}$-Zone, sicher erfasst in der Bohrung Neuenkirchen 2; 
- CM-Zone (claviger-macra) aus dem höheren Tn3 (tieferer Teil der CM-Zone), erfasst in der Bohrung Neuenkirchen 2.

Die zwei entsprechenden Kernmärsche in der Bohrung Wiek 4 (Tabelle 2) sind weniger spezifisch und nehmen vermutlich eine Position etwa im ND/RM-Grenzniveau und eventuell im Hangendbereich der RM-Zone am Übergang zur $\mathrm{CM}-Z$ one ein.

2. Die pusilla-Zone im überregionalen Sinn (Pu-Zone sensu lato) setzt auf Rügen mit dem V1a ein, makrofaunistisch geeicht in der Neuenkirchen 2 und Wiek 4. Der Nachweis von Lycospora pusilla im basalen V1a ist aber so sporadisch (nur Einzelfunde), dass die Brauchbarkeit als alleiniges Kriterium für die Korrelation im Rahmen der regionale Skala infrage zu stellen ist. Für die Korrelation wurde deshalb ein regionales Hilfskriterium - der im V1a anzutreffende Horizont mit Gloeocapsamorpha - genutzt, der in diesem Niveau sowohl in der Neuenkirchen 2 wie auch in der Wiek 4 nachgewiesen ist.

Solange konkret keine Lycospora pusilla nachgewiesen ist, wird die Zonenbezeichnung der $\mathrm{CM}-Z$ Zne in der regionalen Skala beibehalten, obwohl durchaus bereits die Möglichkeit der Zugehörigkeit zur Pu-Zone sensu lato besteht. Die Präzisierung muss dann durch Hilfskriterien erfolgen.

3. Die sporadische Verteilung von L. pusilla im V1a von Rügen ist wie folgt belegt:

Bohrung Neuenkirchen 2: (a) Tiefster Nachweis im basalen V1a? in der CM-Zone $=\mathrm{Pu}-\mathrm{Zo}-$ ne s. 1., noch unterhalb des GloeocapsamorphaNiveaus. (b) Innerhalb des GloeocapsamorphaNiveaus gelang kein Nachweis! CM-Zone $(=\mathrm{Pu}-$ Zone s. l., Zugehörigkeit von b zur Pu-Zone nur durch den Nachweis in a). (c) Eindeutige Nachweise im höheren V1a und V1b?, Pu-Zone.

Bohrung Wiek 4: Tiefster Nachweis von L. pusilla im Glococapsamorpha-Horizont in zwei unterschiedlichen Kernmärschen des V1a. Der Übergang zum Liegenden unterhalb des Gloeocapsamorpha-Horizontes (?mehrere Niveaus) fehlt durch eine Kernlücke.

4. Die von Carson (1995) und Carson \& Clayton (1997) behauptete Diskrepanz zwischen der makrofaunistisch kontrollierten Gliederung nach Lithoeinheiten und der von ihnen sporenstratigraphisch abgeleiteten, abweichenden Lage der Tournai-Visé-Grenze in den Bohrungen Rügen 2 (Abweichung ca. $300 \mathrm{~m}$ ) und Wiek 3 (ca. $450 \mathrm{~m}$ ) kann für die Bohrungen Neuenkirchen 2 und Wiek 4 nicht bestätigt werden - in der Neuenkirchen 2 und Wiek 4 stimmen die makrofau- nistische und sporenstratigraphische Aussage zur Lage der Tournai-Visć-Grenze überein.

5. Die Ursache des Widerspruches wurde von Carson \& Clayton (1997) nicht diskutiert, obgleich seit Burmann (1975: 883) der Nachweis von $L$. pusilla im makrofaunistisch datierten $\mathrm{Vla}$ von Rügen gesichert ist. Es wird vermutet, dass die Suche nach L. pusilla in den tieferen Horizonten der Bohrungen Rügen 2 und Wiek 3 nicht intensiv genug durchgeführt wurde und kein Hilfskriterium als Anhaltspunkt für eine solche Suche abgeleitet wurde.

\section{Danksagung}

Herr Dr. D. Weyer (Berlin) hat nicht unwesentlich dazu beigetragen, dass ich mich der Unterkarbon-Thematik wieder zugewandt habe. Für seine Bemühungen bei der Manuskriptdurcharbeitung sci ihm herzlich gedankt. Auch wurden die biostratigraphischen Datierungen (Details im Teil 2) von ihm durch die makrofaunistischen Angaben ergänzt, sodass diesc damit autorisiert sind. Weitere kollegiale Hinweise verdanke ich Herrn Dr. A. Kampe (Berlin). Herr Prof. Dr. H.-P. Schultze, Direktor des Museums für Naturkunde Berlin, gestattete in der projektlosen Zeit den Zugang zu den Institutseinrichtungen des Institutes für Paläontologie der Humboldt-Universität Berlin und förderte die Manuskripterarbeitung.

\section{Literatur}

Avchimovitch, V. I. 1993. Zonation and sporc complexes of the Devonian and Carboniferous boundary deposits of the Pripyat depression (Byelorussia). - Annales de la Société Géologique de Belgique 115 (1992-2): 425-451.

Avchimovitch, V. I., Byvsheva, T. V.. Higgs. K.. Streel, M. \& Umnova, V. T. 1988. Miospore systematics and stratigraphic correlation of Devonian-Carboniferous boundary deposits in the European part of the USSR and Western Europe. - Courier Forschungsinstitut Senckenberg 100: 169-191

Avchimovitch, V. I. \& Turnau, E. 1994. The Lower Carboniferous Prolycospora claytonii Zone of Western Pomerania and its equivalents in Belorussia and Northwestern Europe. - Annales Societatis Geologorum Poloniac 63: $249-263$.

Avchimovitch. V. I., Turnau, E. \& Clayıon. G. 1993. Correlation of uppermost Devonian and Lower Carboniferous miospore zonations in Byelorussia, Poland and Western Europe. - Annales de la Société Géologique de Belgique 115 (1992-2): 453-458.

Becker, G., Bless. M. J., Streel, M. \& Thorez, J. 1974. Palynology and ostracode distribution in the Upper Devonian and basal Dinantian of Belgium and thcir dependence on sedimentary facies. - Mededelingen Rijks Geologische Dienst, N. S. 25 (2): $9-99$.

Bertelsen, F. 1972. A Lower Carboniferous microflora from the Ørslev No. 1 borehole, Island of Falster, Denmark. Danmarks Geologiske Undersøgelse II. Raekke. Nr. 99 (Geol. Surv. Denmark, II Series. No. 99): 1-78.

Burmann. G. 1975. Sporen aus dem Tournai von Rügen. Zeitschrift für geologische Wissenschaften 3 (7): 875-905. 1976. Sporen und Phytoplankton aus den Devon/KarbonGrenzschichten des Harzes (lepidophytus-Zone). - Zeitschrift für geologische Wissenschaften 4 (6): 805-835. 
Byvsheva. T. V. 974. Zonalnoje rastschlenenije po sporam turneiskich, nishne- $\mathrm{i}$ srednevisejskich otloshenij VolgoUralskoi oblasti. In Palinologija proterofita i paleofita: 100-105. Isda elstvo Nauka. Moskva. [russ.].

Byvsheva. T. V. \& Umnova. N. I. 1993. Palynological characteristics of the lower part of the Carboniferous of the Central R zigion of the Russian Platform. - Annales de la Société Géologique de Belgique 115 (1992-2): $519-529$.

Byvsheva. T. V.. Higgs. K. \& Streel. M. 1984. Spore correlation between the Rhenish Slate Mountains and the Russian Platform near the Devonian-Carboniferous boundary. - Col rier Forschungsinstitur Senckenberg 67: $37-45$.

Carson. B. \& Clavton. G. 1997. The Dinantian (Lower Carboniferous) pelynostratigraphy of Rügen. Northern Germany. - Prace Państwowego Instvtutu Geologicznego 157 (1): $219-227$.

Clayton. G. 1971. A Lower Carboniferous miospore assemblage from the Calciferous Sandstone Measurcs of the Cockburnspatl region of eastern Scotland. - Pollen et Spores 12: $577-600$.

- 1996. Chapter 18C: Mississippian miospores. In Jansonius J. \& McGregcr. D. C. (eds). Palynology: Principles and Applications. American Association of Stratigraphic Palynologists Foun dation (AASP) 2: 589-596.

Clayton. G., Coqu el, R., Doubinger, J.. Gueinn. K. J.. Loboziak. S. Owen. B. \& Streel. M. 1977. Carboniferous miospores of Western Europe: illustration and zonation. Mededelingen Rijks Geologische Dienst 29: 1-71.

Clayton. G. \& Turnau, E. 1990. Correlation of the Tournaisian miospore sonations of Poland and the British Isles. Annales Socielatis Geologorum Poloniae 60: 45-58.

Dolby. G. \& Neves, R. 1970. Palynological evidence concerning the Devonian-Carboniferous Boundary in the Mendips. England. - Compte Rendu fème Congrès International de Stratigraphie et Géologie du Carbonifêre (Sheffield 1967) 2: 631-647.

Filipiak. P. 1997. The Devonian-Carboniferous boundary in the Kowala Trench (Holy Cross Mts. Poland). based on miospores. - 'race Państwowego Instytutu Geologicznego 157 (1): 23 : -241 .

Hacquebard. P. A 1957. Plant spores in coal from the Horton Group (Nississippian) of Nova Scotia. - Micropaleontology $3(-)$ : $301-324$.

Higgs, K. T. 1975. Upper Devonian and Lower Carboniferous miospore assemblage from Hook Head. Co. Wexford. Ireland. - Micropaleontology 21 (4): 393-419.

Higgs. K. T. \& Clayton. G. 1984. Toumaisian miospore assemblage from Maesbury in the eastern Mendips. Fneland. - Journa! of Micropalaeontology 3 (1): 17-28.

Higgs. K.. Claytou. G. \& Keegan. J. B. 1988. The stratigraphic and syste natic palynology of the Tournaisian rocks of Ireland. - (jeological Survey of Ireland. Special Paper 7: $1-93$

Higgs. K.. Dreeser. R.. Dusar. M. \& Streel. M. 1992. Palynostratigraphy of the Tournaisian (Hastarian) rocks in the Namur Svnclinorium. West Flanders. Belgium. - Review of Palaeobotany and Palvnology 72: $149-158$

Higgs. K., McPhil ·my. B., Keegan. J. B. \& Clavton. G. 1988 New data on palynological boundaries within the Irish Dinantian. - Feview of Palaeobotany and Palynology 56 $61-68$.

Higgs. K. \& Stree.. M. 1984. Spore stratigraphy at the Devonian-Carboniferous boundary in the northern "Rheinisches Schiefercebirge“. Germany. - Courier Forschungsinstitut Sencke iberg 67: 157-179.

Higgs, K. Streel. M.. Korn. D. \& Paproth. E. 1993. Palynological data from the Devonian-Carboniferous boundary beds in the new Stockum trench II and the Hasselbach borehole nortiern Rhenish Massif. Germany. - Annales de la Société Géologique de Belgique 115 (1992-2): $551-557$
Hoffmann. N.. Lindert, W., Weyer, D. \& Illers, K.-H. 1975. Zum Unterkarbon-Vorkommen auf den Inseln Rügen und Hiddensee. - Zeitschrift für geologische Wissenschaften 3 (7): 85!-873.

Hoffmeister, W. S.. Staplin, F. L. \& Malloy, R. E. 1955. Mississippian plant spores from the Hardinsburg Formation of Illinois and Kentucky. - Journal of Paleontology 29 (3): $372-399$.

Hughes. N. F. \& Playford, G. 1961. Palynological reconnaisance of the Lower Carboniferous of Spitsbergen. - Micropaleontology 7: $27-44$

Ischtschenko. A. M. 1956. Spores and Pollen of the Lower Carboniferous deposits of the Donetz Basis and their stratigraphic importance. - Trudy Instituta Geologicheskich Nauk Akademii Nauk Ukrainskoi SSR, seria stratigrafija i paleontologija 11: 1-185. [russ.].

Jachowicz. A. 1966. Microfloristic characteristics of the deposits of the Lublin Carboniferous. - Prace Państwowego Instytutu Geologicznego 44: 104-134.

- 1967. Microflora of the Zarȩby beds from Swiętokrzyskie Mountains. - Prace Państwowego Instytutu Geologicznego 49: $1-108$.

- 1970. Tournaisian and Upper Viséan microfloras of the Swięty Krzyż Mountains (Central Poland) their stratigraphical and palaeogeographical value. Compte Rendu 6ème Congrès International de Stratigraphie et Géologie du Carbonifère (Sheffield 1967) 3: 983-1008.

Jäger. H. 2000. Palynostratigraphie und Palynofazies im mittleren Visé von Rügen. - Kurzlassung Poster, 70. Jahrestagung der Paläontologischen Gesellschaft, Coburg.

Kedo, G. I. 1957a. Stratigrafitcheskoje znachenie Hymenozonotriletes pusillites sp. $\mathrm{n}$. - Doklady Akademii Nauk Belorusskoi SSR 1 (1): 21. [russ.].

- 1957b. O stratigrafii i sporovo-pylcevych kompleksach nizhnych gorizontov karbona SSSR. - Doklady Akademii Nauk SSSR 115 (6): 1165-1168. [russ.].

- 1957c. Spory nadsolvych devonskich otlozhenij Pripjatskovo progiba i ich stratigraficheskoje znachenie. In Paleontologija i stratigrafija BSSR 2: 3-43. [russ.].

- 1963. Spory turnejskovo jarusa Pripjatskovo progiba i ich stratigraficheskoje znachenie. In Paleontologija i stratigrafija BSSR 4: 3-121. [russ.]

- 1966. Spory nizhnevo karbona Pripjatskovo progiba. In Paleontologija i stratigrafija Byelorusskoi SSR 5: 1-143. [russ.].

- 1974. Palinologitscheskoje obosnovanije granizy devona i karbona v Pripjatskoi vpadine. In Palinologija proterofita i paleofita: 86-92: Isdatelstvo Nauka, Moskva. [russ.].

Kedo. G. I. \& Golubzov, V. K. 1971. Palinologitscheskij kri terij dlja opredelcnia granizzy devona $\mathrm{i}$ karbona $\vee$ Pripjatskoi vpadine. In Palinologitschekije issledovanija v Bvelorussii i drugich raionach SSSR: 5-35; BelNIGRI, Mezhdunar. Palinolog. Kongr.: Isdatelstvo Nauka i Technika. Minsk. [russ.].

Kedo. G. I.. Nasarenko, A. M., Nekrjata, N. S., Raskatova, L. G. Sennova. V. F. \& Tschibrikova, E. V. 1971. Nowyje vidy spor is famenskich otloshenij Pripjatskoi vpadinv. zentralnich raionov Russkoi platformy, VolgoUralskoi neftegasonosnoi provinzii i Timana. In Palinologitscheskije issledovanija $\vee$ Byclorussii $\mathrm{i}$ drugich raionach SSSR: 172-205; BelNIGRI, Mezhdunarodnij Palinologitscheskij Kongres; Isdatelstvo Nauka i Technika, Minsk. [russ.]

Luber. A. A. \& Walz, I. E. 1938. Classification and stratigraphical importance of spores of some Carboniferous localities in USSR. - Trudy ZNIGRI (Zentralnovo NautschnoIssledovatelskovo Geologo-Rasvedotschnovo Instituta) 105: 1 - 45 . [russ.].

- 1941. Atlas mikrospor i pylzy paleosoja SSSR. - Trudy VSEGEI (Vsesojusnovo Geologicheskovo Instituta) 139. [russ.]: (zitiert nach Kedo 1966)

Matyja. H. \& Turnau. E. 1989. Conodonts and spores from Devonian/Carboniferous boundary beds in Poland. - 
Compte Rendu 11ème Congrès International de Stratigraphic et Géologie du Carbonifère (Beijing 1987) 3: $61-72$.

Maziane, N. \& Vanguestaine, M. 1996. Acritarchs from the uppermost Famennian at Chanxhe and Tohogne (eastern Belgium). - Acta Universilatis Carolinae, Geologica 17: $527-530$

Mariane: N., Higgs, K. T. \& Streel, M. 1999. Revision of the late Famennian miospore zonation scheme in eastern Belgium. - Journal of Micropalaeontology 18 (1): 17-25.

Naumova; S. N. 1953. Spore-pollen complexes of the Upper Devonian of the Russian Platform and their stratigraphic value. - Trudy Instituta geologicheskich Nauk, Akademia Nauk SSSR 143: 1-204. [russ.].

Neves, R., Gueinn, K. J., Clayton, G., Ioannides, N. \& Neville, R. S. W. 1972. A scheme of miospore zones for the British Dinantian. - Compte Rendu 7ème Congrès International de Stratigraphie et de Géologie du Carbonifère (Krefeld 1971) 1: 347-353.

Owens. B. 1983. Bascaudaspora - a new genus. - Institute of Geological Sciences (NERC), Report 83-10: 45-49.

Owens. B. \& Streel, M. 1967. Hymenozonotriletes lepidophytus Kedo, its distribution and significance in relation to the Devonian/Carboniferous boundary. - Review of Palacobotany and Palynology 1: 141-150.

Paproth, E. \& Streel, M. 1971. Corrélations biostratigraphiques près de la limite Dévonien/Carbonifère entre les faciès littoraux ardennais et les faciès bathyaux rhénans. - Colloque sur la Stratigraphie du Carbonifère - Les Congrès et Colloques, Univcrsité Liège 55: 365-398.

Paproth. E., Conil, R., Bless, M. J. M., Boonen, P., Boukkaert, J., Carpentier, N., Coen, M., Delcambre, B., Deprijck, Ch., Deuzon, S., Dreesen, R., Groessens, E., Hance, L., Hennebert, M., Hibo, D., Hahn, G. R., Hislaire, O. Kasig, W., Laloux, M., Lauwers, A., Lees, A., Lys, M., Op de Beek, K., Overlau, P., Pirlet, H., Poty, E., Ramsbottom, W. Streel, M., Swennen, R., Thorez, J., Vanguestaine, M. Van Steenwinkel, M. \& Vieslet, J. L. 1983. Bio- and Lithostratigraphic Subdivisions of the Dinantian in Belgium, A Review. - Annales de la Societé Géologique de Belgique 106: 185-239.

Playford, G. 1962. Lower Carboniferous microfloras of Spitsbcrgen. Part One. - Palaeontology 5 (3): 550-618.

- 1963. Lower Carboniferous microfloras of Spitsbergen. Part Two. - Palaeontology 5 (4): 619-678.

- 1964. Miospores from the Mississippian Horton Group, Eastern Canada. - Bulletin of the Geological Survey of Canada 107: 1 - 47 .

- 1971. Lower Carboniferous spores from the Bonaparte Gulf Basin, Western Australia and Northern Territory. Bulletin of the Burcau of Mineral Resources, Geology and Geophysics (Australia) 115: 1-105.

- 1976. Plant microfossils from the Upper Devonian and Lower Carboniferous of the Canning Basin, Western Australia. - Palaeontographica B 158: 1-71.

- 1991. Australian Lower Carboniferous miospores relevant to extra-Gondwanic correlations: an evaluation. - Courier Forschungsinstitut Senckenberg 130: 85-125.

Richardson, J. B. 1962. Spores with bifurcate processes from the Middle Old Red Sandstone of Scotland. - Palaeonto$\operatorname{logy} 5$ (2): $171-194$.

- 1965. Middle Old Red Sandstone spore assemblage from the Orcadian basin, north-east Scotland. - Palaeontology 7 (4): 559-605.

Schopf, J. M., Wilson, L. R. \& Bentall, R. 1944. An annotated synopsis of Paleozoic fossil spores and the definition of generic groups. - Illinois State Geological Survey, Report Investigation 91: 1-72.

Stockmans, F. \& Willière, Y. 1960. Hystrichosphères du Dévonien belge (sondage de l'Asile d'aliénés à Tournai). Senckenbergiana lethaea 41 (1-6): 1-11.

- 1962. Hystrichosphères du Dévonien belge (sondage de Wépion). - Bulletin de la Société belge de géologie, de paléontologie et d'hydrogeologie 71: 83-99.
- 1964. Addition à la flore du Dévonien supérieur de la Belgique. - Bulletin de la Société belge de géologie, de paléontologie et d'hydrogeologie 72: 371-374.

Streel, M. 1966. Critères palynologiques pour une stratigraphie détaillée du Tnla dans les bassins ardenno-rhénans. - Annales de la Société Géologique de Belgique 89 (3): $65-96$.

- 1969. Corrélations palynologiques entre les sédiments de transition Dévonien/Dinantien dans les bassins ardennorhénans. - Compte Rendu 6ème Congrès International de Stratigraphie et Géologie du Carbonifère (Sheffield 1967) 1: 3-18.

- 1971. Distribution stratigraphique et géographique d'Hymenozonotriletes lepidophytus Kedo, d'Hymeno-(onotriletes pusillites KFDO et des assamblages tournaisiens. - Colloque sur la Stratigraphie du Carbonifère. Les Congrès et Colloques, Université Liège 55: 121-147.

- 1972. Biostratigraphie des couches de transition DévonoCarbonifère et limite entre les deux Systèmes (Synthèse du groupe de travail sur la limite Dévonicn/Carbonifère). - Compte Rendu 7ème Congrès International de Stratigraphie et Géologie du Carbonifère (Krefeld 1971) 1: $167-178$

- 1974. In Becker, G., Bless, M. J. M., Streel, M. \& Thorez, J. 1974. Palynology and ostracode distribution in the Upper Devonian and basal Dinantian of Belgium and their dependence on sedimentary facies. - Mededclingen Rijks Geologische Dienst 25 (2): 9-98.

- 1986. Miospore contribution to the upper Famennian-Strunian event stratigraphy. - Annales de la Société Géologique de Belgique 109: 75-92.

Streel, M., Caputo, M. V., Loboziak, S. \& Melo. J. H. G. 2000. Late Frasnian-Famennian climates based on palynomorph analyses and the question of the Late Devonian glaciations. - Earth-Science Reviews 52: 121-173.

Streel, M., Higgs, K., Loboziak, S. , Riegel, W. \& Steemans. P. 1987. Spore stratigraphy and correlation with faunas and floras in the type marine Devonian of the ArdenneRhenish regions. - Review of Palaeobotany and Palynology 50: $211-229$.

Streel, M. \& Loboziak, S. 1994. Observations on the establishment of a Devonian and Lower Carboniferous highresolution miospore biostratigraphy. - Review of Palaeobotany and Palynology 83: 261-273.

- 1996. Chapter 18B: Middle and Upper Devonian miospores. In Jansonius, J. \& McGregor, D. C. (eds). Palynology: Principles and Applications. American Association of Stratigraphic Palynologists Foundation (AASP) 2: 575-587, Dallas.

Strecl, M., Loboziak, S, Stcemans, P. \& Bullynck, P. 200(). Devonian miospore stratigraphy and correlation with the global stratotype sections and points. - Courier Forschungsinstitut Senckenberg 220: 9-23.

Sullivan, H. J. 1964. Miospores from the Drybrook Sandstone and associated measures in the Forest Dean Basin: Gloucestershire. -- Palaeontology 7: 351-392.

- 1968. A Tournaisian spore flora from the Cementstonc group of Ayrshire, Scotland. - Palaeontology 11 (1): 116-131.

Turnau, E. 1975. Microflora of the Famennian and Tournaisian deposits from boreholes of northern Poland. - Acta Geologica Polonica 25 (4): 505-528.

- 1978. Spore zonation of uppermost Devonian and Lower Carboniferous deposits of Western Pomerania ( $N$ Poland). - Mededelingen Rijks Geologische Dienst 30 (1): $1-35$.

- 1990. Spore zones of Famennian and Tournaisian deposits from the Kowala 1 borehole. - Kwartalnik Geologiczny 34(2): 291 - 304 .

Weyer, D. 2001. Korallen im Unterkarbon Deutschlands. Abhandlungen und Berichte für Naturkunde des Museums Magdeburg 23: 57-91, (dated 2000). 
Wierich. F. \& Vogt. W. 1997. Zur Verbreitung. Biostratigraphie und Petrc graphie unterkarbonischer Sandsteine des Höre-Gommen-Zuges im ostlichen Rhenohercynikum. - Gcologica et Palaeontologica 31: 97-142.

Winslow, M. 1962. Plant spores and other microfossils from Upper Devonic $n$ and Lower Mississippian rocks of Ohio. - United Stat ss Geological Survey. Professional Paper Paper 364: 1-93.

\section{Unveröffentlichte Berichte, Manuskripte und Dissertationen:}

Burmann. G. 1974. Unterkarbon. Mikrobotanische Bearbeitung: 37-71 /n Blumenstengel. H.. Burmann. G.. Döring. H.. Frever. G. Fahlert. E. \& Wever. D. Paläontologie und Biostratigraphic des Oberkarbon. Unterkarbon und Oberdevon der Bohrung E Neuenkirchen 2/1973. - Zentrales Geologis ches Institut Berlin. Bericht Nr. EE 35/ 1974: 1-135 (Archiv BGR Berlin).

- 1971. Untersuct ung organischer Mikrofossilien im Unterkarbon der Bonrung Wiek 4. - Zentrales Geologisches Institut Berlin. Bericht Nr. EE $11 / 71$ vom 22.1.1971 1-23: (Archiv BGR Berlin).

2001. Detailang then zu den Sporenassomiationen (tepids. phyta- bis pusilla-Zone) aus dem Unterkarbon der Bohrungen Neucnkirchen 2/1973 und Wiek 4/1970 (Insel Rü gen. NE-Deutschland). - Zeitschrift für geologischo Wissenschaften 29 (4).

Carson. B. 1995. The palynostratigraphy, palynofacies and hydrocarbon sour :c potential of the Lower Carboniferous in two borehols from the Baltic Island of Rigen. Germany. - Unpublished Ph. D. Dissertation. University of Dublin. Departnent of Geology. Trinity College Dublin.

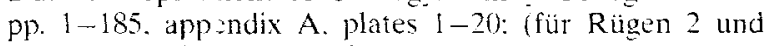
Wiek 3: Archiv BGR Berlin).

Hoffmann. N. Lincert. W. \& Weyer. D. (in Vorbereitung für 2002). Das Unt rkarbon am Südwestrand der Osteuropäischen Plattforn (Inseln Rügen. Hiddensee. Usedom: Mecklenburg-Vorpommern). - Manuskript. Deutsche Subkommission für Karbon-Stratigraphie [füt Courier Forschungsinstit $₫$ Senckenberg].

Jäger. H. 1999. Secimentologie und Biostratigraphie der unterkarbonischen Quarzitfolge der Hörre-Gommern-Zone im Rhenoherzynikum. - Dissertation (Intemet) TU Darmstadt (Fac ibereich Geowissenschaften und Geographie): $1-178$.

\section{Anhang 1. Taxa-Liste}

Der Vermerk (Burmann 1975) bedeutet. dass diese Formen aus dem Tn1 - Tn2-Kernmarsch der Bohrung Wick 4 aus der NRH-Zone von Rigen 1975 abgebildet wurden. Der Vermerk (Burmann 1976) bezieht sich auf Abbildungen zu den Arten aus der var. typica-Subzone (Fazd) der lepidophytaZone aus der südlichen Umrandung des Elbingeröder Komplexes im Mittelhar:

Die Artenliste ir sgesamt bezieht sich aber auf alle drei Vorkommen mit Sp renassoziationen des Tournai und seiner Grenzschichten: in den beiden Bohrungen Wiek 4 und Neuenkirchen 2. d. h. von der lepidophyta-Zonc (var. minor. höheres Tnla) bis zur pusilla-Zone (hier Vla-Vlb). und den Einzelvorkommen der lepidophyta-Zone im Mittelharz.

Die Zitate der A: ten erfolgen hier - im Unterschied zum teilweisc ahgekürzten Verfahien im übrigen Text - in kompletter botanischer Nomenklatur (mit Autor. Kombinator. Jahreszahl).

\section{Sporen}

Acanthotriletes sp. A (in Burmann 1975: Taf. II/9, 10)

Acanthotriletes dominans (Luber \& Walz, 1941) Kedo, 1963

Acanthotriletes mirus Ischtschenko, 1956 (in Burmann 1975: Taf. III/3)

Actanthotriletes multisetus (Luber \& Walz, 1941) Playford, 1962

Archaeomiletes hebetis Kedo, 1963

Archaeo-onotriletes intertextus Nekrjata \& Sergeeva, 1971 (in Kedo et al. 1971)

Auroraspona macra Sullivan, 1968

Auroraspora solisorta Hoffmeister. Staplin \& Malloy, 1955 (in Burmann 1976: Taf. IV/1; 5)

Baculatisporites fusticulus Sullivan, 1968 (in Burmann 1975: Taf. $11 / 6)$

Camptozonotriletes velatus (Waltz, 1938 in Luber \& Waltz, 1941) Playford. 1963

Chaetosphaerites tariabilis Jachowicz, 1970 (in Burmann 1975: Tat. II/7:8)

Comolutispora vermifomis Hughes \& Playford, 1961 (in Burmann 1975: Taf. VIII/2; 7-9)

Convolutispora aff. lermifomis Hughes \& Playford, 1961 (in Burmann 1975: Taf. VIII/1)

Cristatisporites echinatus Playford, 1963

Ciclogramisporites sp. A (sensu Hacqucbard, 1957) (in Burmann 1975: Taf. I/6)

Dicospora multifurcata Winslow. 1962 (in Burmann 1976: Tat. $\mathrm{I} / 1)$

Dictyotriletes asperitis (Kedo, 1963) Burmann, 1975 (in Burmann 1975: Taf. IX/5)

syn.?: Bascalldaspora mischkinansis (Byvsheva) Byvsheva (in Alchimovitch 1993)

Dictrotriletes aff. asperitis (Kedo, 1963) (in Burmann 1975: Taf. IX/4)

Dictromiletes wivialis (Kedo, 1963)

Endosporites minutus Hoffmeister. Staplin \& Malloy, 1955 (in Bumann 1975 Tal. I/ -5)

Grandispinosa conspicala (Playt.) Playford, 1971 (sensu Streel. 1974) (in Burmann 1976: Taf. IV/2)

Grandispora echinata Hacquebard, 1957

Hymenozonotriletes aff. proelegans Kedo, 1963

Knoxisporites hederatus (Ischtschenko, 1956) Playford, 1963 (in Burmann 1975:Taf. VIl/1-6)

Knoxisporites literatus (Waltz, 1938) Playford, 1963, var. triangularis

Lophotriletes circumscriptum Kedo, 1966

Lophozonomileses bellus Kedo, 1963 (in Burmann 1975: Taf. III $/ 4)$

Lophozonotriletes cristifer (Luber \& Wal7. 1941) Kedo, 1963

Lophotriletes minutissimus sensu Kedo, 1963

Lophotriletes tuberosus Burmann, 1975 (in Burmann 1975: Taf. III/2)

Lophotriletes aff. uberosus Burmann, 1975 (in Burmann 1975: Taf. III/1:5)

Lophozonotriletes rarituberculatus (Luber \& Walz, 1941) Kedo. 1957 (in Burmann 1975: Taf. I/7, II/2, 4, IV/1-9)

neu kombiniert: Tumulispora rarituberculata (Luber \& Walz, 1941) Playford. 1991

Lrcospora pusilla (Ibrahim) Schopf, Wilson \& Bentall, 1944

Murospora aurita Playford, 1962

Punclatisporites limbatus Hacquebard, 1957 (in Burmann 1975: Taf. 1/9)

Punctatisporites solidus Hacquebard, 1957 (in Burmann 1975: Taf. I/s)

Raistrickia sp. (in Burmann 1976: Taf. I/2)

Raistrickia clavata (Hacquebard, 1957) Playford. 1964

Raistrickia corynoges Sullivan. 1968

Raistrickia lariabilis Dolby \& Neves, 1970 (in Burmann 1975: Taf. VI/1-2: 4-5)

Raistrickia aff. variabilis Dolby \& Neves, 1970 (in Burmann 1975: Taf. VI/3:6)

Retialetes sp. A (in Burmann 1975: Taf. IX/1-2) 
Reticulatisporites aff. cheveriensis Playford, 1963 (in Burmann 1975: Taf. IX/3)

Retispora lepidophyta (Kedo, 1957) var. typica (in Burmann 1976: Taf. I/3, II/1-3, III/1-2, IV/3c, 4)

Retispora lepidophyta (Kedo, 1957) Playford, 1976 var. minor Rhabdosporites cf. parvalus Richardson, 1965 (s. Streel, 1974) (in Burmann 1976: Taf. IV/3)

Rugospora sp.

Schopfites claviger Sullivan, 1968

Spinozonotriletes uncatus Hacquebard, 1957 (in Burmann 1975: Taf. II/5)

Umbonatisporites distinctus Clayton, 1971

Vallatisporites Hacquebard, 1957

Vallatisporites pusillites (Kedo, 1957) Dolby \& Neves, 1970 s. 1. Vallatisporites verrucosus Hacquebard, 1957

Verrucosisporites nitidus Playford, 1964 (in Burmann 1975: Taf. V/1-3)

syn. Verrucosisporites grumosus (Naumova, 1953) Sullivan, 1964

Verrucosisporites congestus Playford, 1964 (in Burmann 1975: Taf. V/4-6)

Übergang zu V. nitidus (bzw. syn. V. grumosus)

Verrucosisporites papulosus Hacquebard, 1957 (in Burmann 1975: Taf. I/10-12)

Verrucosisporites gobbettii Playford, 1962 (in Burmann 1975: Taf. II/1)

\section{Mikroplankton und Algen}

zahlreiche Kleinformen von Lophosphaeridium sp.

Baltisphaeridium longispinosum Eisenack (in Burmann 1976: Taf. VII/8)

Cavatisporites microreticulatus Jachowicz, 1967

Gloeocapsamorpha prisca Zalesky

Gorgonisphaeridium ohioense Winslow, 1962 (in Burmann 1976: Taf. VI/1-5, VII/2)

Hercyniana meissneri Burmann, 1976 (in Burmann 1976: Taf. $\mathrm{VII} / 1)$

Leiosphaeridia sp. (in Burmann 1976: Taf. V/1-2)

Micrhystridium oceanicum Stockmans \& Willière, 1960 (in Burmann 1976: Taf. VII/5-6)

Tomacia sarjeanti Stockmans \& Willière, 1964 (sensu Streel, 1974)

Veryhachitum helgicum Stockmans \& Willière, 1960 (in Burmann 1976: Taf. VII/7)

Vervhachium downiei Stockmans \& Willière, 1962 (in Burmann 1976: Taf. VII/3)

\section{Anhang 2. Vergleich lepidophyta-Zone}

Der Anhang 2 beinhaltet die Aufbereitung einiger Literaturdaten, die wichtig sind für die Einstufung der in der Bohrung Neuenkirchen 2/1973 auf Rügen sowie im Harz (Burmann 1976) angetroffenen Sporenassoziation der lepidophyta-Zone.

Aus der umfangreichen Literatur zur Sporenzonierung im Devon-Karbon-Grenzbereich und Tournai sei auf einige ausgewählte Arbeiten verwiesen:

- für das Ardenno-Rhenische Becken: Streel $(1966,1986)$, Stockmans \& Willière $(1960,1962,1964)$, Paproth \& Streel (1971), Becker et al. (1974), Paproth et al. (1983), Streel et al. (1987, 2000), Higgs \& Streel (1984), Higgs et al. (1992, 1993), Maziane \& Vanguestaine (1996), Maziane ct al. (1999)

- für Westeuropa: Sullivan (1968). Dolby \& Neves (1970), Higgs (1975), Higgs \& Clayton (1984), Higgs et al. (1988)

- für Rügen und den Harz: Burmann $(1975,1976)$, Carson (1995), Carson \& Clayton (1997)

- für Polen, die Pripjat-Senke und Osteuropa: Luber \& Walz $(1938,1941)$, Naumova (1953), Ischtschenko (1956), Kedo (1957a-c, 1963, 1966, 1974), Kedo \& Golubzov (1971), Kedo et al. (1971), Jachowicz (1966, 1967, 1970),
Byvsheva (1974), Byvsheva \& Umnova (1993). Byvsheva et al. (1984). Turnau (1975, 1978, 1990). Matyja \& Turnau (1989), Clayton \& Turnau (1990), Filipiak (1997), Avchimovitch (1993), Avchimovitch et al. (1988. 1993, 1994)

- für die Sporenzonierung: Neves et al. (1972), Higgs \& Streel (1984), Clayton (1996), Streel \& Loboriak (1994. 1996), Streel et al. (2000).

Vorkommen in Mitteleuropa: Im mitteldeutschen Raum (Rügen und Harz) sind bisher aus der lepidophyta-Zone die biometrischen Subzonen var. minor (Neuenkirchen 2. Tab. 1; Burmann 1975) und var. typica (Harz. Burmann 1976) erfasst. Das Vorkommen in der Bohrung Neuenkirchen 2 ist durch Weyer (unpubliziert) makrofaunistisch als Etroeungt (Strunium, Tn1a) sensu lato datiert, wobei die Untergrenze nicht sicher bestimmbar ist (auch $\mathrm{Fa} 2 \mathrm{~d}$ ist nicht auszuschließen). Die sonst fossilfreien Schicfer mit $R t^{\prime}$ tispora lepidophyta im Harz sind nur durch die Sporen einstufbar.

Die Sporenführung der Vorkommen auf Rügen und im Harz lässt aber einc differenziertere Aitersaussage zu. Alle Fundorte müssen als isolierte Vorkommen gewertet werden - es besteht kein direkter Übergang zu einer im Liegenden oder Hangenden anschließenden Sporenzone, bei der Bohrung Neuenkirchen 2 wird das Vorkommen durch Kernmarschlücken (der nächsttiefere Kernmarsch ist fossilfrei) begrenzt, im Harz sind es einzclne Fundpunktnachweise in einem tektonisch gestörten Schiefergebiet. Eine Zuordnung nach Indexfossilien, die in der Regel auf dem Ersteinsetzen von Indexarten setzt (Maziane et al. 1999), ist bei den isolierten, profilmäßig begrenzten Vorkommen deshalb schwer zu realisieren. Als praktikabel hat sich aber für dic stratigraphische Zuordnung der isolierten Vorkommen die biometrische Subzonengliederung nach Streel 1966 erwiesen. Auf dieser Grundlage ist für das Vorkommen in der Bohrung Neuenkirchen 2 eine Zuordnung zur lepidophyta var. minor-Subzone (biometrische Zone $\mathrm{F}$; $\mathrm{d}=42 \mu \mathrm{m}$ Optimum) und damit eine Einstufung in das höhere Tnla möglich. Das Vorkommen von var. minor ist auf die biometrischen Subzonen D. E und $F$ beschränkt. Die Zugehörigkeit der vorliegenden Form zur Subzone F geht hervor aus dem Fehlen von var. typica und var. tener sowie aus der Präsenz von Lophozonotriletes rarituberculatus und Verrucosisporites nitidus als Komponenten der PLs1-Subzone (Einsetzen der Formen).

Sporenstratigraphische Korrelation: In den übrigen Profilen von Rügen wurde bisher keine dem Kernmarsch 2090,0-2098,5 m der Bohrung Neuenkirchen 2 vergleichbare Sporenassoziation angetroffen; das Niveau liegt nur noch einmal (Bohrung Dranske 2) im Bohrkern vor. Außerhalb Rügens bieten sich gute Korrelationsmöglichkeiten sowohl zum Ardenno-Rhenischen Raum wie auch insbesondere zur Pripjat-Senke. Der Ardenno-Rhenische Raum steht dabei nur deshalb stärker im Blickpunkt. weil hier die biometrische Zonenfolge innerhalb der lepidophvta-Zone (Streel 1966) abgeleitet wurde. Beim ersten Nachweis der lepidophyta-Zone im Harz (Burmann 1976) wurde indirekt schon Bezug genommen auf das hier beschriebene Vorkommen von Rügen; die Vergleichsbasis wird nachfolgend diskutiert.

Definition der biometrischen Zonen nach Streel (1966): Die Definition der biometrischen Zonen A-F innerhalb der lepidophyta-Zone beruht auf Unterschieden im Sporendurchmesser (Tendenz zur Größenabnahme vom Liegenden zum Hangenden und zunehmende Auflösung der charakteristischen Netzstruktur) sowie auf der Berücksichtigung des Einsetzens der Häufigkeitsmaxima und des Verschwindens der verschiedenen Varietäten (var. typica, var. tener, var. minor). Die biometrische Zonenfolge ist in drei kombinierten, regional eng zusammenhängenden Profilen definiert worden (Streel 1966): Chanxhe (A-E), Rivage gare (A-E, ? F), Modave (F). Die Grenzen zwischen den Zonen $\mathrm{C}-\mathrm{D}-\mathrm{E}$ sind im Chanxhe-Profil definiert in den Niveaus 112 (C-D) und 129 (D-E), die Zone F im Niveau Modave 47. 
Tabelle 6

Biometrische Glie lerung der lepidophyta-Zone

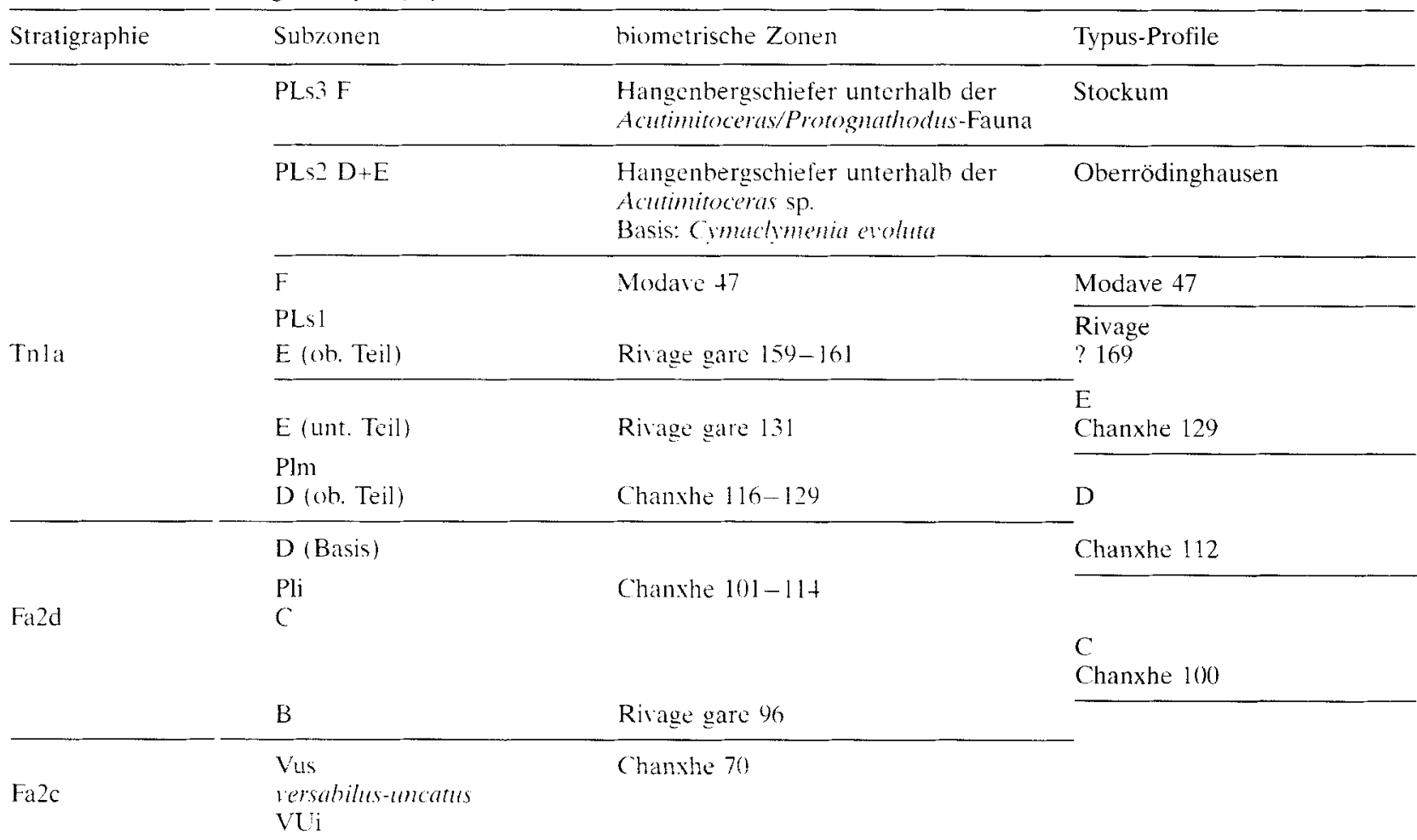

Parallel zur bionnetrischen Zonengliederung wurde später (Paproth \& Streel 1971: 374: Streel 1972: 174) von Streel in diesen Profilen eine Subzonengliederung der lepidophvtu-Zone in die Subzonen PLi. PL.m. PLsl aufgestellt. an die aus isolierten Profilen die in ihrer Selbstständigkeit umstrittenen Subzonen PLs2 (Oberrödinghausen) und PLs3 (Stockum) angeschlossen wurdon. Dabei stimmen die Subzonengrenzen nicht mit den Gronzen der biometrischen Zonen überein (Tab. 6).

Korrekturen zu dieser Sporenzonen-Gliederung sind in Maziane et al. (1909: 5) enthalten: sie bezichen sich auf die LE- (lepidophyta-explahatus) und die LI-Zone (lepidophytaliteratus) des Tn1a. Die Basis des Fa2d ist definiert durch das Einsetzen von Retspora lepidophwa (Maziane 1999: 23): nahe bei deren Ers erscheinen setzt nach den neuen Daten auch Knoxisporites literatus ein, sodass der Umfang der früheren LL-Zone rum Liegenden erweitert wurde. Die biometrischen Zonen von Tab. 6 können über dic Subzonenglicderung nach Paproth \& Streel (1971) in Bezug gesetzt werden zur Gliederung nach Indexfossilien (Maziane et al. 1999) in Tab. 7.

Die biometrischen Zonen der lepidophyta-Zone definiorte Strecl (1966: 76, 1969):

Zone F: Fehlen von Großformen der var. typica; Vorkommen von var. minor a. $40-50$ um; b. 36-46 um; Häufigkeitsoptimum 40-46 fm (Vergleich Tnla Bohrung Ncuenkirchen 2 - Häufigkeitsoptimum $42 \mu \mathrm{m}$ )

Zone F: Fehlen von Großformen der var. typica $40-50 \mu \mathrm{m}$ (entspricht var. minor). Häufigkeitsoptimum $46-50 \mu \mathrm{m}$

Tabelle 7

Sporenzonen an der Deron/Karbon-Grence

\begin{tabular}{|c|c|c|c|}
\hline Stratigraphie & $\begin{array}{l}\text { Paproth \& Streel } 1971 \\
\text { Becker et al. } 1974\end{array}$ & $\begin{array}{l}\text { Paproth et al. } 1983 \\
\text { Streel \& Loboziak } 1996\end{array}$ & $\begin{array}{l}\text { Maziane ct al. } 1999 \\
\text { Präzisierung }\end{array}$ \\
\hline $\begin{array}{l}\text { Unterkarbon: } \\
\text { Tnlb }\end{array}$ & & VI-Zone vallatus-incohatus & \\
\hline \multirow[t]{2}{*}{$\begin{array}{l}\text { Oberdevon: } \\
\text { Tnla }\end{array}$} & & \multicolumn{2}{|l|}{ LN-Zone 21 lepidophyta-nitidus } \\
\hline & & LE-Zone 20 lepidophyta-explanatus & LE \\
\hline & $\begin{array}{l}\text { PL pusillites-lepidophytus: } \\
\mathrm{Pls} 2\end{array}$ & LL-Zone lepidophya-literatus & \\
\hline $\mathrm{Fa} 2 \mathrm{~d}$ & $\begin{array}{l}\text { Pls } 1 \\
\text { Plm } \\
\text { Pli }\end{array}$ & LV-Zone 19 lepidophyta-verrucosa & LL \\
\hline $\mathrm{Fa} 2 \mathrm{c}$ & $\begin{array}{l}\text { VL } \\
\text { versabilis-ancants }\end{array}$ & VCo-Zone $16-18$ versabilis-cornuta & $\begin{array}{l}\text { VH verrucosa-hystr } \\
\text { VCo versabili-scom }\end{array}$ \\
\hline
\end{tabular}


Zone D: noch wenige Großformen der var. typica; daneben Häufigkeitsoptimum 56-60 $\mu \mathrm{m}$ (var. tener); (46-50 $\mu \mathrm{m}$ var. minor)

Zone C: Häufigkeitsmaximum von var. typica $>70 \mu \mathrm{m}$ (60)-66 $\mu \mathrm{m}, 70-76 \mu \mathrm{m})$

Zone B: Einsetzen von wenigen Exemplaren der var. typica Zone A: Fehlen der Art.

Die Varietäten von Hymenozonotriletes lepidophytus werden von Kedo \& Golubzow (1971: 27-30) wie folgt angegeben:

\begin{tabular}{lll}
\hline Varietät & Gesamtdurchmesser & Zentralkörper \\
\hline var. typica & $55-90 \mu \mathrm{m}$ & $45-55 \mu \mathrm{m}$ \\
var, tener & $55-76 \mu \mathrm{m}$ & $29-45 \mu \mathrm{m}$ \\
var. minor & $42-54(44) \mu \mathrm{m}$ & $30-40 \mu \mathrm{m}$
\end{tabular}

Der Inhalt der Subzonen PLi, PLm, PLs1, PLs2, PLs3 der lepidophyta-Zone war in Streel (1971) und Streel in Becker et al. (1974) nur in etwa aus den fragmentarischen Sporenlisten für die Typusprofile Chanxhe und Rivage gare ( $\mathrm{Pa}-$ proth \& Streel 1971: 374-375) und den stratigraphischen Verbreitungstabellen zu entnehmen. Danach wäre die Basis der PLs1-Zone (höherer Teil Zone E Rivage gare 159-161) durch das Einsetzen von Lophozonotriletes rarituberculatus bei gleichzeitigem Vorkommen von var. minor charakterisiert. Diesem Kriterium des gleichzeitigen Vorkommens beider Komponenten entspricht auch die Assoziation im Tn1aKernmarsch der Bohrung Neuenkirchen 2, der damit der PLs1 (bzw. PLs)-Subzone entspricht, die im Wesentlichen den höchsten Teil der biometrischen Zone $\mathrm{E}$ und die gesamte Zone $\mathrm{F}$ umfasst.

Folgende mit dem Rügen-Profil vergleichbare Assoziationen sind im Ardenno-Rhenischen Becken beschrieben ( $\mathrm{Pa}$ proth \& Streel 1971):

Rivage gare 159-161 - PLs1 (E) mit Hymenozonotriletes lepidophytus, Lophozonotriletes rarituberculatus, Raistrickia variabilis, Dicrospora multifurcata, Grandispora echinata

Ratingen 17-19 - PLs1 (E und F) mit Hymenozonotriletes lepidophytus, Lophozonotriletes rarituberculatus, Grandispora echinata, Knoxisporites $\mathrm{cf}$. hederatus

Modave 47 - PLsI (F)

Yvoir-tunnel 41 - PLs1 (F) mit Hymenozonotriletes lepidophytus, Lophozonotriletes rarituberculatus, Verrucosisporites nitidus, Grandispora echinata, Raistrickia variabilis, Endosporites ex gr. minutus, Auroraspora macra, Knoxisporites cf. hederatus

Etrocungt 3-4 - PLs1 mit Hymenozonotriletes lepidophytus, Lophozonotriletes rarituberculatus, Cristatisporites echinatus, Endosporites ex gr. minutus, Grandispora echinata, Raistrickia variabilis

Hönnctal PLs2 (D-E) und Stockum (F) PLs3 - Hangenbergschiefer mit Hymenozonotriletes lepidophytus, Lophozonotriletes rarituberculatus, Verrucosisporites nitidus, Auroraspora macra, Vallatisporites pusillites, Grandispora echinata, Knoxisporites literatus, Cristatisporites echinatus.

Es ist gegenwärtig nicht zu entscheiden, inwieweit die var. minor-Subzone der lepidophyta-Zone eventuell geringfügig noch in das Tn1b hineinreicht, da entsprechende Eichungsmöglichkeiten an anderen Fossilgruppen fehlen. Auch ist der direkte Übergang des obersten Teils der PL-Zone (F) in die hangende TE (trivinlis-explanatus)-Zone des $\mathrm{Tn} 1 \mathrm{~b}$ nirgends beschrieben worden; Strecl (1972: 169) deutete derartige Mogglichkeiten für das Stockum-Vorkommen erst an. Alle si-
Tabelle 8

Vergleich Rügen und Bjelorussia

Strati- Bohrung Neuenkirchen Pripjat-Senke
graphie $2 / 1973$

\begin{tabular}{lll}
\hline Tn1b & $\begin{array}{l}2069,8-2078,5 \mathrm{~m} \\
\text { pusillites-Zone }\end{array}$ & $\begin{array}{l}\text { untere Malev-Schichten } \\
\text { ml1-Komplex } \\
\text { pusillites-Zone }\end{array}$ \\
\hline & $\begin{array}{l}\text { höheres Tn1a: } \\
2090,0-2098,5 \mathrm{~m} \\
\text { var. minor-Subzone } \\
\text { (biometr. Zone F) }\end{array}$ & $\begin{array}{l}\text { Oser-Chowan-Schichten } \\
\text { IIIB-Komplex }\end{array}$ \\
\cline { 2 - 3 } Tn1a & & $\begin{array}{l}\text { Dankowo-Lebedjan-Scht. } \\
\text { oberster Teil: } \\
\text { IIIA-Komplex (Einsetzen } \\
\text { von L. rubituberculatus) }\end{array}$ \\
& $\begin{array}{l}\text { Belgien: Basis der } \\
\text { PLs-Zone (E) }\end{array}$ & $\begin{array}{l}\text { Dankowo-Lebedjan-Scht. } \\
\text { höherer Teil: II-Komplex }\end{array}$ \\
\hline Fa2d & $\begin{array}{l}\text { Belgien: Äquivalent } \\
\text { Zone C }\end{array}$
\end{tabular}

cheren Nachweise für Retispora lepidophyta var. minor beschränken sich jedoch bisher auf das höhere Tnla.

Im Gegensatz zum Ardenno-Rhenischen Gebiet sind die aus der Pripjat-Senke (Weißrussland) bekannten Sporenassoziationen im Devon/Karbon-Grenzbereich kaum an Faunengruppen zu eichen. Die aus dem überwiegend terrigenen Profil beschriebenen Mikrofloren haben jedoch den großen Vorzug, dass sie jeweils in eine Profil-Abfolge eingefügt werden können. Diese Möglichkeit ergab sich aus zahlreichen Bohrungen dieser Region (Kedo 1957, 1963, 1966, 1974. Kedo \& Golubzow 1971).

Die Sporenassoziation aus dem Tnla-Kernmarsch der Bohrung Neuenkirchen 2 ist innerhalb dieser Abfolge von Sporenassoziationen aus der Pripjat-Senke mit dem IIIBKomplex der Ozer-Chowan-Schichten vergleichbar. Allerdings setzt Lophozonotriletes rarituberculatus bcreits im IIIA-Komplex des obersten Teils der Dankowo-LebedjanSchichten ein, einem Abschnitt, in dem Retispora lepidophyta var. typica zurücktritt und die Varietäten var. minor, var. tener, Hymenozonotriletes cassis vorherrschen. Die Korrelation mit den biometrischen Zonen Streels erscheint etwas problematisch, obwohl die Tendenz der Größenreduzierung und der Vertikalverteilung der Varietäten von Kedo (1974: 87) in Analogie zu den belgischen Verhältnissen bestätigt wird. Die Vergleichbarkeit zwischen dem Tn1a-Kernmarsch der Bohrung Neuenkirchen 2 und dem IIIB-Komplex der Ozer-Chowan-Schichten (Kedo \& Golubzow 1971) wird durch die groBe Formenmannigfaltigkeit des Letzteren bestätigt. Weitere Aussagen müssten jedoch Detailuntersuchungen vorbehalten bleiben. Die bisherige Vergleichsmöglichkeit im Devon-Karbon-Grenzbereich zeigt Tab. 8 .

Als gemeinsame pflanzengeographische Besonderheit ist zu vermerken, dass in der Bohrung Neuenkirchen 2 und in der Pripjat-Senke Vallatisporites pusillites (sensu lato) erst in der pusillites-Zone des 'Tn1b auftritt, während die Art im Ardenno-Rhenischen Becken und in anderen Regionen in der gesamten lepidophyta-Zone verbreitet ist. Der Gegensatz zwischen dem Ardenno-Rhenischen Becken und der PripjatSenke ist in Bezug auf diese Komponente extrem: Dies muss wohl als pflanzengeographische Besonderheit gewertet werden 